\title{
ON A SPECTRAL SEQUENCE FOR EQUIVARIANT K-THEORY
}

\author{
MARC LEVINE, CHRISTIAN SERPÉ
}

\begin{abstract}
We apply the machinery developed by the first-named author to the $K$-theory of coherent $G$-sheaves on a finite type $G$-scheme $X$ over a field, where $G$ is a finite group. This leads to a definition of $G$-equivariant higher Chow groups (different from the Chow groups of classifying spaces constructed by Totaro and generalized to arbitrary $X$ by Edidin-Graham) and an AtiyahHirzebruch spectral sequence from the $G$-equivariant higher Chow groups to the higher $K$-theory of coherent $G$-sheaves on $X$. This spectral sequence generalizes the spectral sequence from motivic cohomology to $K$-theory constructed by Bloch-Lichtenbaum and Friedlander-Suslin.
\end{abstract}

\section{Contents}

1. Introduction 1

2. Equivariant $G$ and $K$-theorv 3

3. The equivariant homotopy coniveau tower 9

4. Local coefficients and the cvcle class map 11

4.1. The local coefficients 12

4.2. Functorialities 14

4.3. The cycle map 16

5. Localization and reduction to the point 17

6. The case of the point 21

References 25

\section{INTRODUCTION}

There are many different approaches to a theory of equivariant algebraic $K$ theory; for simplicity, we restrict our discussion to the case of a finite group $G$ acting on a scheme $X$. One can use algebraic versions of the Borel construction, taking an sequence of finite dimensional approximations $U_{n}$ to $E G$ and then defining $K_{*}^{G}(X)$ as a limit of the $K_{*}\left(\left(U_{n} \times X\right) / G\right)$. This approach gives a different answer depending on the underlying topology chosen; for the Zariski topology, one may use the standard simplicial model of $E G$ whereas for the étale topology it is necessary to use other models. This latter choice gives for example an equivariant $K_{0}$-group closely related to the equivariant Chow groups first defined by Totaro [Tot99] in the case of $X$ a point, generalized to arbitrary $X$ by Edidin-Graham. EG98, and

Date: October 10, 2018.

1991 Mathematics Subject Classification. Primary 19E15; Secondary 14C99, 14C25.

The first-named author gratefully acknowledges the support of the Humboldt Foundation through the Wolfgang Paul Program, and support of the NSF via grants DMS-0140445 and DMS0457195 . 
studied by Edidin-Graham [EG00, Pandharipande [Pan98] and many others. One can also modify this approach by using the homotopy fixed-point spectrum $K\left(U_{n} \times\right.$ $X)^{G}$ instead of the $K$-theory of the quotient.

Another approach is to take the $K$-theory spectrum of the exact category of locally free $G$-sheaves on $X$, or the $G$-theory analog using coherent $G$-sheaves. We will henceforth denote these two theories by $K_{*}(G, X)$ and $G_{*}(G, X)$. These theories differ in general from the ones described above, mainly due to the lack of étale descent for algebraic $K$-theory.

It is possible to give an allied cycle theory by using the orbit category of $G$, as in topological Bredon (co)homology. This approach has been used by Joshua Jos05, and has yielded for example Riemann-Roch type results for $G_{*}(G, X)$.

We will use a somewhat different approach. We feed the equivariant $G$-theory spectra $G(G, X)$ into the homotopy coniveau machine developed in Lev01, Lev05. What comes out is a theory of algebraic cycles with an interesting "local coefficient" system, together with an associated theory of higher Chow groups and most importantly, a spectral sequence

$$
E_{p, q}^{1}=C H_{p}(G, X, p+q) \Longrightarrow G_{p+q}(G, X) .
$$

In the case $G=\{\mathrm{id}\}$, this reduces to the Bloch-Lichtenbaum/Friedlander-Suslin spectral sequence BL95, FS02.

The local coefficient system is easy to describe: Let $Y$ be a finite type $k$-scheme with a $G$-action, $Z \subset Y$ an irreducible closed subset with generic point $z$. Let $G_{z} \subset G$ be the isotropy group of $z$. Then $G_{z}$ acts on the residue field $\kappa(z)$, and we define the "coefficient group" of $Z$ to be $K_{0}\left(G_{z}, \kappa(z)\right)$. The dimension $p$ equivariant cycle group of $Y$ is then

$$
z_{p}(G, Y):=\oplus_{[z] \in\left(Y_{(p)}\right) / G} K_{0}\left(G_{z}, \kappa(z)\right) .
$$

To explain: $Y_{(p)}$ is the set of points $z \in Y$ with closure $\bar{z}$ having dimension $p$ over $k$. $G$ acts on $Y_{(p)}$ and $z$ is a representative of the orbit $[z] \in\left(Y_{(p)}\right) / G$.

Now if $X$ is a finite type $k$-scheme with $G$-action, taking the dimension $p+$ $r$ points of $X \times \Delta^{r}$ in "good position" with respect to the faces of $\Delta^{r}$, as in Bloch's construction of his cycle complexes Blo86, and using our coefficient system $[z] \mapsto K_{0}\left(G_{z}, \kappa(z)\right)$, one defines the Bredon-type equivariant cycle complex $z_{p}(G, X, *)$ and equivariant higher Chow groups $C H_{p}(G, X, *)$. The arguments of Lev01, Lev05 go through without much change to yield the spectral sequence (11).

As alluded to above, one can view our construction as giving a Bredon-type motivic Borel-Moore homology theory for the quotient stack $[X / G]$. It seems reasonable that one could extend the construction of the cycle theory and the spectral sequence to more general stacks, either by removing the restriction that $G$ is finite (e.g. allowing $G$ to be a linear algebraic group) or considering stacks other than quotient stacks. It would also be interesting to see if Joshua's construction, applied to the Friedlander-Suslin-Voevodsky cycle complexes, yield the same motivic Borel-Moore homology groups as ours.

Part of the development of this paper was made possible by the hospitality of the University of Duisberg-Essen, the University of Münster and the Wolgang Paul program of the Alexander von Humboldt Foundation. We would like to express our sincere gratitude for this support. 


\section{Equivariant $G$ AND $K$-THEORY}

Let $G$ be a finite group and $X$ be a (left) $G$-scheme. A coherent $G$-module $\mathcal{F}$ on $X$ is a coherent $\mathcal{O}_{X}$-module together with an action of $G$ on this module which is compatible with the action of $G$ on $X$. In other words, for each $g \in G$ we have a morphism $\phi_{g}: g^{*} \mathcal{F} \rightarrow \mathcal{F}$ and for $g, h \in G$ we have $\phi_{g \cdot h}=\phi_{h} \circ h^{*} \phi_{g}$. We denote by $\mathcal{M}_{G, X}$ the abelian category of coherent $G$-modules and by $\mathcal{P}_{G, X}$ the exact subcategory of $\mathcal{M}_{G, X}$ of those G-modules which are locally free as $\mathcal{O}_{X}$-modules. We further denote by $G(G, X)$ and $K(G, X)$ the K-theory spectrum of $\mathcal{M}_{G, X}$ resp. $\mathcal{P}_{G, X}$. Then $G(G, X)$ is contravariant with respect to flat $G$-morphisms in $X$ and covariant with respect to projective $G$-morphisms in $X . K(G, X)$ is contravariant with respect to any $G$-morphism.

If $X=\operatorname{Spec} R$ for a commutative ring $R$ the (left) action of $G$ on $X$ induces a (right) action of $G$ on the ring $R$. We set $R^{t w}[G]:=\oplus_{g \in G} R e_{g}$. By

$$
\left(r_{g} \cdot e_{g}\right)\left(r_{h} \cdot e_{h}\right):=r_{g} \cdot\left(r_{h} \cdot g^{-1}\right) e_{(g \cdot h)}
$$

for all $g, h \in G$ and $r_{g}, r_{h} \in R$ we define a ring structure on $R^{t w}[G]$ and we call $R^{t w}[G]$ the twisted group ring of $R$.

Lemma 2.1. Let $X=\operatorname{Spec} R$ be a noetherian affine $G$-scheme. Then the category of finitely generated $R^{\text {tw }}[G]$-modules is equivalent to $\mathcal{M}_{G, X}$.

Proof. For a $R$-module $M$ and $g \in G$ let ${ }_{g} M$ be the $R$-module which is $M$ as abelian group and where the $R$-module structure is defined by $r \cdot{ }_{g} M m:=\left(r \cdot g^{-1}\right) \cdot m$ for all $r \in R$ and $m \in M$. A $G$-module on Spec $R$ is the same as an $R$-module together with $R$-module homomorphisms $\phi_{g}:{ }_{g} M \rightarrow M$ for all $g \in G$ with $\phi_{(g h)}=\phi_{h} \circ \phi_{g}$ for all $g, h \in G$. Then $M$ becomes a left $R^{t w}[G]$-module if we set $e_{g} \cdot m:=\phi_{g^{-1}}(m)$. Conversely if $M$ is a $R^{t w}[G]$-module we define $\phi_{g}:{ }_{g} M \rightarrow M$ by $\phi_{g}(m):=e_{g^{-1}} \cdot m$ for all $g \in G$ and get in this way a $G$-module on Spec $R$. Obviously the two functors are inverse to other. Because $G$ is finite and $R$ noetherian the lemma follows.

Lemma 2.2. Let $R$ be commutative noetherian ring with $\frac{1}{\sharp G} \in R$. Then the category of finitely generated projective $R^{t w}[G]$-modules is equivalent to $\mathcal{P}_{G, \operatorname{Spec} R}$.

Proof. If $\sharp G$ is invertible in $R$ then each $R^{t w}[G]$-module which is projective as $R$ module is also projective as $R^{t w}[G]$-module. So the lemma follows from the lemma above.

Now let $k$ be a fixed commutative ring with $\frac{1}{\sharp G} \in k$. We denote by $\mathbf{k}$-alg the category of commutative $k$-algebras. Let $F: \mathbf{k}$-alg $\rightarrow \mathbf{A b}$ be an additive functor. Following Vorst Vor79, we define

$$
\begin{array}{cccc}
N F: \quad \mathbf{k - a l g} & \rightarrow & \mathbf{A b} \\
R & \mapsto & k e r(F(R[T]) \rightarrow F(R)),
\end{array}
$$

where the last map is induced by $R[T] \rightarrow R, T \mapsto 0$. For $q>1$ we define inductively $N^{q} F:=N\left(N^{q-1} F\right)$.

For $f \in R$ the morphism $R[X] \rightarrow R[X], X \mapsto f \cdot X$ induces a group endomorphism $N F(R) \rightarrow N F(R)$. So $N F(R)$ becomes a $\mathbb{Z}[T]$ module. We denote by $N F(R)_{[f]}$ the $\mathbb{Z}\left[T, T^{-1}\right]$ module $\mathbb{Z}\left[T, T^{-1}\right] \otimes_{\mathbb{Z}[T]} N F(R)$. With these notations Vorst proves the following theorem. 
Theorem 2.3. Let $R \in \boldsymbol{k}$-alg and let $r_{1}, \ldots, r_{n}$ be elements of $R$ which generate the unit ideal. Suppose further that the map

$$
N F\left(R[T]_{r_{i_{0}}, \ldots, r_{\hat{i}_{j}}, \ldots, r_{i_{p}}}\right)_{\left[r_{i_{j}}\right]} \rightarrow N F\left(R[T]_{r_{i_{0}}, \ldots, r_{i_{p}}}\right)
$$

is an isomorphism, for each set of indexes $1 \leq i_{0}<\cdots<i_{p} \leq n$. Then the canonical morphism

is injective.

$$
\epsilon: N F(R) \rightarrow \oplus_{j=1}^{n} N F\left(R_{r_{j}}\right)
$$

Proof. Compare [Vor79 Theorem 1.2] or Lev94 Lemma 1.1].

As in [TT90, one may form the Bass delooping $K B(G, X)$ of the -1-connected spectrum $K(G, X)$. The functor $X \mapsto K B(G, X)$ satisfies

(1) There is a canonical map $K(G, X) \rightarrow K B(X, G)$, identifying $K(G, X)$ with is the -1-connected cover of $K B(G, X)$.

(2) For every $X$, there is the natural exact sequence

$$
\begin{aligned}
0 \rightarrow K B_{p}(G, X) \rightarrow K B_{p}\left(G, X \times \mathbb{A}^{1}\right) & \oplus K B_{p}\left(G, X \times \mathbb{A}^{1}\right) \\
& \rightarrow K B_{p}\left(G, X \times \mathbb{G}_{m}\right) \rightarrow K B_{p-1}(G, X) \rightarrow 0
\end{aligned}
$$

called the fundamental exact sequence.

(3) If $X$ is regular, then $K(G, X) \rightarrow K B(G, X)$ is a weak equivalence.

From now on, we will drop the notation $K B(G, X)$ and write $K(G, X)$ for the (possibly) non-connected version.

Now let $X=\operatorname{Spec} C$ be an affine $G$-scheme over $k$. We consider for each $p \in \mathbb{N}$ the functor

$$
\begin{array}{cccc}
K_{p}\left(G, X \otimes_{k}-\right): & \mathbf{k}-\mathbf{a l g} & \rightarrow & \mathbf{A b} \\
R & \mapsto & K_{p}\left(G, X \otimes_{k} R\right),
\end{array}
$$

where $G$ acts trivially on $R$. We say that $X$ is $K_{p}(G,-)$-regular if $N^{q} K_{p}(G, X)=0$ for all $q>0$.

Lemma 2.4. Let $R \in \boldsymbol{k}$-alg and $f \in R$. Suppose that there is an $g \in R$ such that $f g=0$ and $f+g$ is a non-zero divisor. Then the natural map

$$
N^{q} K_{p}\left(G, X \otimes_{k} R\right)_{[f]} \rightarrow N^{q} K_{p}\left(G, X \otimes_{k} R_{f}\right)
$$

is an isomorphism.

Proof. By Lemma 2.2 we have the identification

$$
K_{p}\left(G, X \otimes_{k} R\right)=K_{p}\left(\left(C \otimes_{k} R\right)^{t w}[G]\right) .
$$

Furthermore the element $f \in C \otimes_{k} R$ lies in the center of the ring. The proof of Lemma 1.4 in Vor79 then goes through word for word to prove the lemma.

Theorem 2.5. Let $R \in \boldsymbol{k}$-alg be reduced and $f_{1}, \ldots, f_{n} \in R$ such that $\left(f_{1}, \ldots, f_{n}\right)=$ $R$ and such that $X \otimes_{k} R_{f_{i}}$ is $K_{p}(G,-)$-regular for $i=1, \ldots, n$. Then $X \otimes_{k} R$ is also $K_{p}(G,-)$-regular.

Proof. This follow from theorem 2.3 and lemma 2.4

Corollary 2.6. Let $X=\operatorname{Spec} C$ be a reduced affine $G$-scheme of finite type over a field and let $U_{i} \subset X, i=1, \ldots, n$ an affine $G$-invariant (i.e. $G U_{i} \subset U_{i}$ ) open covering of $X$. Then $X$ is $K_{p}(G,-)$-regular if and only if $U_{i}$ is $K_{p}(G,-)$-regular for $i=1, \ldots, n$. 
Proof. Let $X / G:=\operatorname{Spec} C^{G}$ be the quotient $X$ by $G$ and $\pi: X \rightarrow X / G$ the projection. Then $\pi\left(U_{i}\right)$ are open subsets of $X / G$ and since the $U_{i}$ 's are $G$-invariant we have $\pi^{-1} \pi\left(U_{i}\right)=U_{i}$ (see for example Gro71] [V.1.1]). For each $i=1, \ldots, n$ we write $\pi\left(U_{i}\right)$ as a union of the principle open subschemes

$$
\pi\left(U_{i}\right)=\cup_{j}(X / G)_{f_{i j}}
$$

for suitable $f_{i 1}, \ldots, f_{i m_{i}} \in C^{G}$. Noting that $(X / G)_{f_{i j}}=\operatorname{Spec}\left(C_{f_{i j}}^{G}\right)$ and letting $U_{i j}:=X \otimes_{C^{G}}\left(C_{f_{i j}}^{G}\right)$, we have $U_{i}=\bigcup_{j} U_{i j}$. We apply lemma 2.4 and theorem 2.5 with $k=C^{G}$ to get the result.

In the last section of this paper we need to consider the equivariant $K$-theory of some singular schemes. This can be partially understood by comparing with multi-relative $K$-theory and $K H$-theory, which we now recall.

For an $G$-invariant subscheme $Y$ of a scheme $X$ we define the relative equivariant K-theory spectrum as

$$
K(G, X ; Y):=\operatorname{hofib}(K(G, X) \rightarrow K(G, Y)) .
$$

More generally, for a family $\left\{Y_{1}, \ldots, Y_{n}\right\}$ of $G$-invariant subschemes we inductively define

$$
\begin{aligned}
K\left(G, X ; Y_{1}, \ldots, Y_{n}\right) & := \\
& \operatorname{hofib}\left[K\left(G, X ; Y_{1}, \ldots, Y_{n-1}\right) \rightarrow K\left(G, Y_{n} ; Y_{1} \cap Y_{n}, \ldots, Y_{n-1} \cap Y_{n}\right)\right] .
\end{aligned}
$$

It can easily be seen that this definition is independent of the order of $\left\{Y_{1}, \ldots, Y_{n}\right\}$.

For an affine $G$-scheme $X=\operatorname{Spec} R$ we denote by

$$
K H(G, X):=K H\left(R^{t w}[G]\right)
$$

where on the right hand side $K H$ denotes the homotopy K-theory of Weibel for (not necessarily commutative!) rings (compare Wei89]).

If $\left\{Y_{1}, \ldots, Y_{n}\right\}$ is a family of $G$-invariant affine subschemes of the affine $G$-scheme $X$ we define as above $K H\left(G, X ; Y_{1}, \ldots, Y_{n}\right)$.

There is a natural transformation of functors from rings to spectra

$$
K(-) \rightarrow K H(-)
$$

this induces a natural transformation of functors from rings with a $G$-action to spectra

$$
K(G,-) \rightarrow K H(G,-) .
$$

Since $K(G, T) \rightarrow K H(G, T)$ is a weak equivalence for $T$ regular, the map

$$
K\left(G, X ; Y_{1}, \ldots, Y_{n}\right) \rightarrow K H\left(G, X ; Y_{1}, \ldots, Y_{n}\right)
$$

is a weak equivalence if $X$ and all the intersections $Y_{i_{1}} \cap \ldots \cap Y_{i_{s}}$ are regular.

Also, if $Y=\cup_{i=1}^{n} Y_{i}$, then it is easy to see that

$$
K(G, X ; Y)=K(G, X ; Y, Y)=\ldots=K(G, X ; Y, \ldots, Y) .
$$

and similarly for $K H(G,-)$. The inclusions $Y_{i} \rightarrow Y$ thus induce the maps

$$
\alpha: K(G, X ; Y)=K(G, X ; Y, \ldots, Y) \rightarrow K\left(G, X ; Y_{1}, \ldots, Y_{n}\right)
$$


and

$$
\beta: K H(G, X ; Y)=K H(G, X ; Y, \ldots, Y) \rightarrow K H\left(G, X ; Y_{1}, \ldots, Y_{n}\right) .
$$

By from [Wei89] th. 1.3,Cor. 2.2], $\beta$ is a weak equivalence.

For the reader's convenience, we include a proof of the following elementary fact:

Lemma 2.7. Let $S$ be a ring (not necessarily commutative). Let $I_{1}, I_{2} \subset S$ be twosided ideals, and let $S_{i}:=S / I_{j} S, j=1,2$ and $S_{12}:=S /\left(I_{1}+I_{2}\right) S$. Let $\pi_{i}: S \rightarrow S_{i}$, $\pi_{12, i}: S_{i} \rightarrow S_{12}$ be the quotient maps and let

$$
S_{1} \times S^{S_{12}} S_{2}=\left\{\left(s_{1}, s_{2}\right) \in S_{1} \times S_{2} \mid \pi_{12,1}\left(s_{1}\right)=\pi_{12,2}\left(s_{2}\right)\right\} .
$$

Suppose that the map

$$
\begin{aligned}
& \pi: S \rightarrow S_{1} \times_{S_{12}} S_{2} \\
& \pi(s):=\left(\pi_{1}(s), \pi_{2}(s)\right)
\end{aligned}
$$

is an isomorphism. Suppose in addition that the surjection $S_{2} \rightarrow S_{12}$ is split by a ring homomorphism $\sigma_{2}: S_{12} \rightarrow S_{2}$. Then the sequence

$$
0 \rightarrow K_{1}(S) \stackrel{\left(\pi_{1 *}, \pi_{2 *}\right)}{\longrightarrow} K_{1}\left(S_{1}\right) \times K_{1}\left(S_{2}\right) \stackrel{\pi_{12,1 *}-\pi_{12,2 *}}{\longrightarrow} K_{1}\left(S_{12}\right) \rightarrow 0
$$

is exact.

Proof. We consider the $K_{1}$-sequence. We first show that $\left(\pi_{1 *}, \pi_{2 *}\right)$ is injective. If $\alpha \in \mathrm{GL}_{N}(S)$ goes to zero in $K_{1}\left(S_{1}\right) \times K_{1}\left(S_{2}\right)$ then we can write

$$
\begin{aligned}
& \pi_{1}(\alpha)=\prod_{r=1}^{n} e_{i_{r}, j_{r}}^{\lambda_{r}} \in \mathrm{GL}_{M}\left(S_{1}\right), \\
& \pi_{2}(\alpha)=\prod_{r=1}^{n} e_{i_{r}, j_{r}}^{\mu_{r}} \in \mathrm{GL}_{M}\left(S_{2}\right)
\end{aligned}
$$

for some $M \geq N$ and for some $\lambda_{r} \in S_{1}, \mu_{r} \in S_{2}$ (we may take some $\lambda_{r}$ or $\mu_{s}$ zero if need be). Letting $\bar{\lambda}_{r}=\pi_{12,1}\left(\lambda_{r}\right)$ and $\bar{\mu}_{r}=\pi_{12,1}\left(\mu_{r}\right)$, we have the relation

$$
\prod_{r=n}^{1} e_{i_{r}, j_{r}}^{-\bar{\mu}_{r}} \prod_{r=1}^{n} e_{i_{r}, j_{r}}^{\bar{\lambda}_{r}}=1
$$

in $\mathrm{GL}_{M}\left(S_{12}\right)$. Thus the element of the Steinberg group $S t_{M}\left(S_{12}\right)$ given by

$$
\prod_{r=n}^{1} x_{i_{r}, j_{r}}^{-\bar{\mu}_{r}} \prod_{r=1}^{n} x_{i_{r}, j_{r}}^{\bar{\lambda}_{r}}
$$

defines an element $x \in K_{2}\left(S_{12}\right)$. Lift $x$ to the element

$$
x_{2}:=\sigma_{*}(x)=\prod_{r=n}^{1} x_{i_{r}, j_{r}}^{-\tilde{\mu}_{r}} \prod_{r=1}^{n} x_{i_{r}, j_{r}}^{\tilde{\lambda}_{r}}
$$

in $K_{2}\left(S_{2}\right)$; here $\tilde{\lambda}_{r}=\sigma\left(\bar{\lambda}_{r}\right)$ and similarly for $\tilde{\mu}_{r}$. 
Lift each $\bar{\mu}_{r}$ to an element $\rho_{r} \in S_{1}$. Then

$$
\begin{aligned}
& \pi_{1}(\alpha)=\prod_{r=1}^{n} e_{i_{r}, j_{r}}^{\rho_{r}} \prod_{r=n}^{1} e_{i_{r}, j_{r}}^{-\rho_{r}} \prod_{r=1}^{n} e_{i_{r}, j_{r}}^{\lambda_{r}} \\
& \pi_{2}(\alpha)=\prod_{r=1}^{n} e_{i_{r}, j_{r}}^{\mu_{r}} \prod_{r=n}^{1} e_{i_{r}, j_{r}}^{-\tilde{\mu}_{r}} \prod_{r=1}^{n} e_{i_{r}, j_{r}}^{\tilde{\lambda}_{r}}
\end{aligned}
$$

Clearly the pairs $\left(\rho_{r}, \mu_{r}\right),\left(-\rho_{r},-\tilde{\mu}_{r}\right),\left(\lambda_{r} \tilde{\lambda}_{r}\right)$ define elements $a_{r}, b_{r}$ and $c_{r}$ in $S_{1} \times S_{12}$ $S_{2}=S$ and we have

$$
\alpha=\prod_{r=1}^{n} e_{i_{r}, j_{r}}^{a_{r}} \prod_{r=n}^{1} e_{i_{r}, j_{r}}^{b_{r}} \prod_{r=1}^{n} e_{i_{r}, j_{r}}^{c_{r}},
$$

as desired.

The exactness at $K_{1}\left(S_{1}\right) \times K_{1}\left(S_{2}\right)$ is easier: if $\pi_{12,1 *}\left(\alpha_{1}\right)=\pi_{12,2 *}\left(\alpha_{2}\right)$ in $K_{1}\left(S_{12}\right)$ for $\alpha_{i} \in \operatorname{GL}_{N_{i}}\left(S_{i}\right), i=1,2$, then there is an element $e \in E_{N}\left(S_{12}\right)$ with

$$
\pi_{12,1 *}\left(\alpha_{1}\right)=\pi_{12,2 *}\left(\alpha_{2}\right) e
$$

in $\operatorname{GL}_{N}\left(S_{12}\right)$ for some $N \geq N_{1}, N_{2}$. We can lift $e$ to an $e_{2} \in E_{N}\left(S_{2}\right)$; replacing $\alpha_{2}$ with $\alpha_{2} e_{2}$, we may assume that $N_{1}=N_{2}=N$ and

$$
\pi_{12,1 *}\left(\alpha_{1}\right)=\pi_{12,2 *}\left(\alpha_{2}\right)
$$

in $\operatorname{GL}_{N}\left(S_{12}\right)$. Since $S=S_{1} \times{ }^{S_{12}} S_{2}$, there is a unique $\alpha \in \operatorname{GL}_{N}(S)$ with $\pi_{i}(\alpha)=\alpha_{i}$, $i=1,2$.

The exactness at $K_{1}\left(S_{12}\right)$ follows by using the splitting $\sigma_{*}$ to $\pi_{12,2 *}$.

Let

$$
\Delta^{n}:=\operatorname{Spec} k\left[t_{0}, \ldots, t_{n}\right] / \sum_{i} t_{i}-1 .
$$

Let $\partial_{i} \Delta^{n}$ be the closed subscheme of $\Delta^{n}$ defined by $t_{i}=0$ and $\partial \Delta^{n}=\cup_{i=0}^{n} \partial_{i} \Delta^{n}$, . For a $k$-scheme with $G$-action $X$, we have the product schemes $X \times \Delta^{n}, X \times \partial \Delta^{n}$, with $G$ acting by the identity on $\Delta^{n}, \partial \Delta^{n}$, and by the given action on $X$.

Lemma 2.8. Let $k$ be a field, $G$ a finite group with $\frac{1}{\sharp G} \in k, X$ a regular affine $G$-scheme over $k$, and let $U$ be a $G$-stable affine open subscheme of $X \times \partial \Delta^{n}$. Then $U$ is $K_{p}(G,-)$-regular for all $p \leq 1$.

Proof. It suffices to show that $U$ is $K_{1}$-regular: we have for each $p$ and $q$ the fundamental exact sequence

$$
\begin{aligned}
0 \rightarrow N^{q} K_{p}(G, U) & \\
\rightarrow N^{q} K_{p}(G, U & \left.\times{ }_{k} \mathbb{A}^{1}\right) \oplus N^{q} K_{p}\left(G, U \times_{k} \mathbb{A}^{1}\right) \\
& \rightarrow N^{q} K_{p}\left(G, U \times_{k} \mathbb{G}_{m}\right) \rightarrow N^{q} K_{p-1}(G, U) \rightarrow 0
\end{aligned}
$$

where $G$ acts trivially on $\mathbb{A}^{1}, \mathbb{G}_{m}$. Clearly, if $U$ is $K_{p}(G,-)$ regular, so is $U \times \mathbb{A}^{1}$; by Lemma 2.4 this implies $U \times \mathbb{G}_{m}$ is also $K_{p}(G,-)$ regular. The exact sequence shows that $U$ is $K_{p-1}(G,-)$ regular.

Let $\partial_{\leq m} \mathbb{A}^{n}$ be the closed subscheme of Spec $k\left[x_{1}, \ldots, x_{n}\right]$ defined by $\prod_{i=1}^{m} x_{i}=0$. Note that each point of $U$ has an open neighborhood isomorphic (as a $G$-scheme) to an open neighborhood of $X \times \partial_{\leq m} \mathbb{A}^{n}$ for some $m$. By Corollary 2.6] it suffices to show that $X \times \partial_{\leq m} \mathbb{A}^{n}$ is $K_{1}(G,-)$ regular. We prove this by induction on $n$ and $m$, 
the case of arbitrary $n$ and $m=1$ following from the smoothness of $\partial_{\leq 1} \mathbb{A}^{n}=\mathbb{A}^{n-1}$ over $k$.

Write $R_{n, m}=k\left[x_{1}, \ldots, x_{n}\right] / \prod_{i=1}^{m} x_{i}$. Note that

$$
\partial_{\leq m} \mathbb{A}^{n}=\partial_{\leq m-1} \mathbb{A}^{n} \cup_{\partial_{\leq m-1} \mathbb{A}^{n-1}} \mathbb{A}^{n-1}
$$

and the inclusion $\partial_{\leq m-1} \mathbb{A}^{n-1} \rightarrow \partial_{\leq m-1} \mathbb{A}^{n}$ is split by the projection

$$
\left(x_{1}, \ldots, x_{n}\right) \mapsto\left(x_{1}, \ldots, x_{m-1}, x_{m+1}, \ldots, x_{n}\right),
$$

Thus, for each affine $G$-scheme $Y=\operatorname{Spec} A$ over $k$, we have the ring

$$
S:=A^{t w}[G] \otimes_{k} R_{n, m}
$$

with two-sided ideals $I_{1}=\left(\prod_{i=1}^{m-1} x_{i}\right), I_{2}=\left(x_{m}\right)$ which satisfy the hypotheses of Lemma 2.7. Note also that $S / I_{1}=A^{t w}[G] \otimes_{k} R_{n, m-1}$ and $S / I_{2}=A^{t w}[G] \otimes_{k} R_{n, 1}$, so the sequence

$$
\begin{aligned}
0 \rightarrow K_{1}\left(G, Y \times \partial_{\leq m} \mathbb{A}^{n}\right) & \\
& \rightarrow K_{1}\left(G, Y \times \partial_{\leq m-1} \mathbb{A}^{n}\right) \oplus K_{1}\left(G, Y \times \mathbb{A}^{n-1}\right) \\
& \rightarrow K_{1}\left(G, Y \times \partial_{\leq m-1} \mathbb{A}^{n-1}\right) \rightarrow 0
\end{aligned}
$$

is exact. Taking $Y=X \times \mathbb{A}^{q}$ and using our induction hypothesis shows that $X \times \partial_{\leq m} \mathbb{A}^{n}$ is $K_{1}$-regular.

Proposition 2.9. Let $k$ be a field, $G$ a finite group with $\frac{1}{\sharp G} \in k, X$ a regular affine $k$-scheme with a $G$-action, and $U$ a $G$-stable affine open subscheme of $X \times \Delta^{n}$. Let $\partial U:=X \times \partial \Delta^{n} \cap U, \partial_{i} U:=U \cap X \times \partial_{i} \Delta^{n}$. Then the map

$$
\alpha: K_{0}(G, U ; \partial U) \rightarrow K_{0}\left(G, U ; \partial U_{0}, \ldots, \partial U_{n}\right)
$$

is an isomorphism.

Proof. Both $U$ and all the intersections $\partial U_{i_{1}} \cap \ldots \cap \partial U_{i_{s}}$ are regular, so

$$
K\left(G, U ; \partial U_{0}, \ldots, \partial U_{n}\right) \rightarrow K H\left(G, U ; \partial U_{0}, \ldots, \partial U_{n}\right)
$$

is a weak equivalence. Since

$$
\beta: K H(G, U ; \partial U) \rightarrow K H\left(G, U ; \partial U_{0}, \ldots, \partial U_{n}\right)
$$

is a weak equivalence, it suffices to show that

$$
K_{0}(G, U ; \partial U) \rightarrow K H_{0}(G, U ; \partial U)
$$

is an isomorphism.

We have the commutative diagram, with rows homotopy fiber sequences:

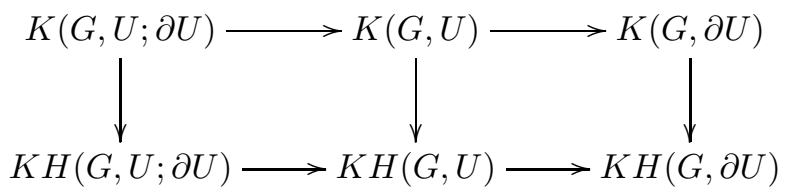

and

$$
\begin{aligned}
& K_{0}(G, U) \rightarrow K H_{0}(G, U) \\
& K_{1}(G, U) \rightarrow K H_{1}(G, U)
\end{aligned}
$$


are isomorphisms, so it suffices to show that the natural maps

$$
\begin{aligned}
K_{0}(G, \partial U) & \rightarrow K H_{0}(G, \partial U) \\
K_{1}(G, \partial U) & \rightarrow K H_{1}(G, \partial U)
\end{aligned}
$$

are isomorphisms. This follows from the weakly convergent spectral sequence (Wei89 th. 1.3])

$$
E_{p, q}^{1}=N^{p} K_{q}(G,-) \Rightarrow K H_{p+q}(G,-)
$$

and the $K_{p}(G,-)$ regularity of $\partial U$ for $p \leq 1$ (Lemma 2.8).

\section{The EqUivariant HOMOTOPY CONIVEAU TOWER}

In this section, we define $G$-equivariant versions of the homotopy coniveau tower defined in Lev01.

Let $G$ be a finite group and $T$ be a $G$-scheme. For a closed $G$-stable subset $W \subset T$ we define

$$
G^{W}(G, T):=\operatorname{hofib}(G(G, T) \rightarrow G(G, T \backslash W)) .
$$

The localization sequence for equivariant $G$-theory gives us the canonical isomorphism

$$
G(G, W) \rightarrow G^{W}(G, T) .
$$

We have as well the $K$-theory version:

$$
K^{W}(G, T):=\operatorname{hofib}(K(G, T) \rightarrow K(G, T \backslash W)) .
$$

and the canonical map

$$
K^{W}(G, T) \rightarrow G^{W}(G, T)
$$

which is a weak equivalence in case $T$ is regular.

We proceed to discuss the functoriality of these constructions. There is always a technical problem occuring at this point, in that the pull-back morphisms on the various exact categories involved are not strictly functorial, but only functorial up to a natural isomorphism satisfying a cocycle condition. Quillen has explained in Qui73 how to rectify this situation by replacing the category of locally free coherent sheaves on a scheme $T$ with an equivalent category, for which there exist strictly functorial pull-back maps. We will use this construction throughout, suppressing its explicit mention, so that the $K$-theory and $G$-theory spectra become strictly functorial constructions.

Let $f: T^{\prime} \rightarrow T$ be a $G$-equivariant morphism of $G$-schemes, $W^{\prime} \subset T^{\prime}$ and $W \subset T$ $G$-stable subsets with $f^{-1}(W) \subset W^{\prime}$. The commutative diagram

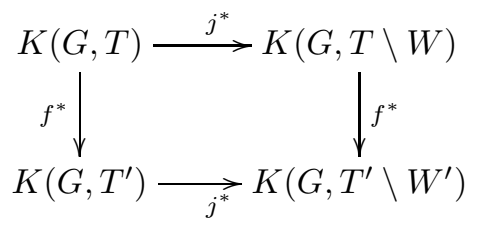

defines the pull-back map $f^{*}: K^{W}(G, T) \rightarrow K^{W^{\prime}}\left(G, T^{\prime}\right)$, satisfying the functoriality $(f g)^{*}=g^{*} f^{*}$. The same holds for $G$-theory if $f$ is flat; in fact, if we require that $f$ factors as $p \circ i$, with $p: \mathbb{P}^{N} \times T \rightarrow T$ the projection and $i: T^{\prime} \rightarrow \mathbb{P}^{N} \times T$ a $G$-equivariant regular embedding into an open subscheme $U$ of $\mathbb{P}^{N} \times T$, then it 
is not hard to show that $f^{*}: G^{W}(G, T) \rightarrow G^{W^{\prime}}\left(G, T^{\prime}\right)$ exists in this case as well. Indeed, $p$ is smooth, so it suffices to define $i^{*}$, and for this, the condition on $i$ and Quillen's resolution theorem show that the inclusion of the $i$-flat $G$-coherent sheafs on $\mathbb{P}^{N} \times T, \mathcal{M}_{G, \mathbb{P}^{N} \times T, i}$, into $\mathcal{M}_{G, \mathbb{P}^{N} \times T}$ induces a weak equivalence on the $K$-theory spectra. See Lev01] for details in the non-equivariant case; the arguments for the equivariant case are exactly the same. A morphism $f$ which admits a factorization $p \circ i$ as above is called an l.c.i.-morphism.

If $T=T^{\prime}$ and $f=\mathrm{id}$, then $\mathrm{id}^{*}$ defines the functorial push-forward map

$$
i_{W^{\prime}, W *}: G^{W}(G, T) \rightarrow G^{W^{\prime}}(G, T)
$$

for $W \subset W^{\prime} G$-stable closed subsets of $T$.

We recall the cosimplicial $k$-scheme $\Delta^{*}, n \mapsto \Delta^{n}$; the cosimplicial structure is defined by sending an order-preserving map $g:\{0, \ldots, n\} \rightarrow\{0, \ldots, m\}$ to the map $g: \Delta^{n} \rightarrow \Delta^{m}$ with

$$
g^{*}\left(t_{i}\right):=\sum_{j \in g^{-1}(i)} t_{j}
$$

Definition 3.1. (1) For $X$ of finite type over $k$, set

$$
S_{(p)}^{G, X}(r):=\left\{\begin{array}{c}
W \text { is a closed } G \text {-stable subset } \\
W \subset X \times \Delta^{r} \mid \\
\text { and for all faces } F \subset \Delta^{r} \text { we have } \\
\operatorname{dim} W \cap X \times F \leq p+\operatorname{dim} F .
\end{array}\right\}
$$

(2) For $X$ finite type and locally equi-dimensional over $k$,

$$
S_{G, X}^{(p)}(r):=\left\{\begin{array}{c}
W \text { is a closed } G \text {-stable subset } \\
W \subset X \times \Delta^{r} \mid \\
\text { and for all faces } F \subset \Delta^{r} \text { we have } \\
\operatorname{codim}_{X \times F} W \cap X \times F \geq p
\end{array}\right\}
$$

The maps $r \mapsto S_{G, X}^{(p)}(r), r \mapsto S_{(p)}^{G, X}(r)$ define simplicial sets; if $X$ has pure dimension $d$ over $k$, we have

$$
S_{G, X}^{(p)}(r)=S_{(d-p)}^{G, X}(r)
$$

We have natural inclusions $S_{G, X}^{(p+1)}(r) \subset S_{G, X}^{(p)}(r), S_{(p)}^{G, X}(r) \subset S_{(p+1)}^{G, X}(r)$.

Definition 3.2. Let $X$ be a $G$-scheme of finite type over $k$. Let

$$
G_{(p)}(G, X, r):=\underset{W \in S_{(p)}^{G, X}(r)}{\operatorname{hocolim}} G^{W}\left(G, X \times \Delta^{r}\right) .
$$

If $X$ is smooth over $k$, let

$$
K^{(p)}(G, X, r):=\underset{W \in S_{G, X}^{(p)}(r)}{\operatorname{hocolim}} K^{W}\left(G, X \times \Delta^{r}\right)
$$

Since $\Delta^{n}$ is smooth over $k$, the structure morphisms in the cosimplicial scheme $X \times \Delta^{*}$ are all l.c.i.-morphisms. Thus, the simplicial structure of $S_{(p)}^{G, X}\left({ }_{-}\right)$gives us the simplicial spectrum $G_{(p)}\left(G, X,{ }_{-}\right)$. In case $X$ is locally equi-dimensional over $k$ we have as well the simplicial spectrum $G^{(p)}\left(G, X,{ }_{-}\right)$and if $X$ is smooth over $k$, the simplicial spectrum $K^{(p)}\left(G, X,_{-}\right)$with term-wise weak equivalence

$$
K^{(p)}\left(G, X,{ }_{-}\right) \rightarrow G^{(p)}(G, X,-)
$$


We let $\operatorname{dim} X$ denote the maximum of $\operatorname{dim} X_{i}$ over the irreducible components $X_{i}$ of $X$. The inclusion $S_{(p)}^{G, X}(r) \subset S_{(p+1)}^{G, X}(r)$ gives rise to the homotopy coniveau tower of simplicial spectra

$$
\cdots \rightarrow G_{(p)}\left(G, X,_{-}\right) \rightarrow G_{(p+1)}\left(G, X,_{-}\right) \rightarrow \cdots \rightarrow G_{(\operatorname{dim} X)}\left(G, X,,_{-}\right)
$$

We denote the layers of this tower by

$$
G_{(p / p-1)}\left(G, X,_{-}\right):=\operatorname{hocofib}\left(G_{(p-1)}\left(G, X,_{-}\right) \rightarrow G_{(p)}\left(G, X,_{-}\right)\right)
$$

Remark 3.3. Since $X \times \Delta^{r}$ is the final element of $S_{(\operatorname{dim} X)}^{G, X}(r)$, the canonical $\operatorname{map} G\left(X \times \Delta^{r}\right) \rightarrow G_{(\operatorname{dim} X)}(X, r)$ is a weak equivalence. By homotopy invariance (compare [Tho87 Cor. 4.2] and Tho87, Th. 5.7]), the map of the constant simplicial spectrum $G(G, X)$ to $G_{(\operatorname{dim} X)}\left(G, X,_{-}\right)$induced by the identity $G(G, X)=G_{(\operatorname{dim} X)}(X, 0)$ is a weak equivalence.

With that notation we have the following proposition.

Proposition 3.4. There is a strongly convergent spectral sequence

$$
E_{1}^{p, q}=\pi_{-p-q}\left(G_{(p / p-1)}\left(G, X,_{-}\right)\right) \Rightarrow G_{-p-q}(G, X)
$$

Proof. The spectral sequence is the spectral sequence of the homotopy coniveau tower (2) together with the identitfication $G_{n}(G, X) \cong \pi_{n} G_{(\operatorname{dim} X)}(G, X$, $)$ given by Remark (3.3). Since

$$
G_{(p)}(G, X, r)=0
$$

for all $r<-p$ and each $G_{(p)}(G, X, r)$ is -1-connected, $G_{(p)}(G, X,-)$ is - $p-1$ connected, and hence the canonical map

$$
G_{(\operatorname{dim} X)}\left(G, X,_{-}\right) \rightarrow \operatorname{hocofib}\left[G_{(p)}\left(G, X,_{-}\right) \rightarrow G_{(\operatorname{dim} X)}\left(G, X,_{-}\right)\right]
$$

is $-p-1$-connected, whence the convergence.

In case $X$ is smooth over $k$, we have the homotopy coniveau tower for equivariant $K$-theory

$$
\cdots \rightarrow K^{(p+1)}\left(G, X,_{-}\right) \rightarrow K^{(p)}\left(G, X,_{-}\right) \rightarrow \cdots \rightarrow K^{(0)}\left(G, X,{ }_{-}\right)
$$

with layers

$$
K^{(p / p+1)}\left(G, X,_{-}\right):=\operatorname{hocofib}\left(K^{(p+1)}\left(G, X,_{-}\right) \rightarrow K^{(p)}\left(G, X,{ }_{-}\right)\right) .
$$

and the strongly convergent spectral sequence

$$
E_{1}^{p, q}=\pi_{-p-q}\left(K^{(p / p+1)}\left(G, X,_{-}\right)\right) \Rightarrow K_{-p-q}(G, X) .
$$

\section{LOCAL COEFFICIENTS AND THE CYCLE CLASS MAP}

In this section, we define the equivariant cycle complex of Bredon type, $z_{q}(X, G, *)$, and use the homology of $z_{q}(X, G, *)$ to define the equivariant higher Chow groups of Bredon type. 
4.1. The local coefficients. For a $k$-scheme $T$, we let $T_{(p)}$ denote the set of points $t \in T$ whose closure $\overline{\{t\}}$ has $\operatorname{dim}_{k} \overline{\{t\}}=p$.

Let $G$ and $X$ be as the the last section. For any smooth $k$-scheme $Y$ we consider the $G$-scheme $X \times Y$, where $G$ acts trivially on the second component. Because $G$ is finite $G$ acts also on the point $(X \times Y)_{(p)}$ of dimension $p$ on $X \times Y$. For an orbit $[x] \in(X \times Y)_{(p)} / G$ of the $G$-set $(X \times Y)_{(p)}$, take a representative $x \in(X \times Y)_{(p)}$ and let $G_{x} \subset G$ denote the isotropy subgroup for $x$. We have the "local coefficient":

$$
\begin{aligned}
\underset{U \subset \overline{G \cdot x}}{\operatorname{colim}} G_{0}(G, U) & =G_{0}\left(G, \coprod_{y \in G \cdot x} \operatorname{Spec}(\kappa(y))\right. \\
& =G_{0}\left(G_{x}, \operatorname{Spec}(\kappa(x))\right) \\
& =K_{0}\left(G_{x}, \operatorname{Spec}(\kappa(x))\right) .
\end{aligned}
$$

Here the colimit on the left hand side is taken over all open $G$-invariant subsets $U$.

We have the following functorial properties for the local coefficients.

Proposition 4.1. Let $[x] \in(X \times Y)_{(p)} / G$ and let $f: Y^{\prime} \rightarrow Y$ be a morphism in $\mathrm{Sm} / k$ of pure codimension $q$. Suppose that

$$
\operatorname{dim}\left(1_{X} \times f\right)^{-1}(\overline{G \cdot x})=p-q .
$$

Then there is a well defined pull back morphism

$$
f^{*}: K_{0}\left(G_{x}, \operatorname{Spec} \kappa(x)\right) \rightarrow \bigoplus_{y \in\left(X \times Y^{\prime}\right)_{(p-q)} / G, f(y) \in G \cdot x} K_{0}\left(G_{y}, \operatorname{Spec} \kappa(y)\right)
$$

with $(f g)^{*}=g^{*} f^{*}$ when all three maps are defined.

Proof. The proof is a modification of Quillen's proof of Gersten's conjecture Qui73. Let $\mathcal{M}^{1}(x)$ be the category of coherent $G$-sheaves on $\overline{G \cdot x}$ whose support contains no generic point, and set $G_{n}^{(1)}(G, \overline{G \cdot x}):=K_{n}\left(\mathcal{M}^{1}(x)\right)$. Consider the localization sequence

$$
\ldots \rightarrow G_{0}^{(1)}(G, \overline{G \cdot x}) \rightarrow G_{0}(G, \overline{G \cdot x}) \rightarrow G_{0}(G, G \cdot x) \rightarrow 0 .
$$

Since $1 \times f$ has finite Tor-dimension, we have the well-defined and functorial map

$$
(1 \times f)^{*}: G_{0}(G, \overline{G \cdot x}) \rightarrow G_{0}\left(G,(1 \times f)^{-1}(\overline{G \cdot x})\right) ;
$$

it clearly suffices to show that the composition

$$
\begin{aligned}
G_{0}^{(1)}(G, \overline{G \cdot x}) \rightarrow G_{0}(G, \overline{G \cdot x}) & \\
& \stackrel{(1 \times f)^{*}}{\longrightarrow} G_{0}\left(G,(1 \times f)^{-1}(\overline{G \cdot x})\right) \rightarrow G_{0}(G, G \cdot y)
\end{aligned}
$$

is the zero map.

If $f: Y^{\prime} \rightarrow Y$ is smooth, and $\mathcal{F}$ is in $\mathcal{M}^{1}(x)$, then $(1 \times f)^{*} \mathcal{F}$ is in $\mathcal{M}^{1}(y)$, whence the result in this case. Also, if $k$ is finite, the standard trick of passing to infinite extensions $k \rightarrow k_{1}, k \rightarrow k_{2}$, with $k_{i}$ a union of extensions of degree $\ell_{i}^{r}$ for distinct primes $\ell_{1}, \ell_{2}$, and using the fact that $p_{*} \circ p^{*}=\times m$ on $G_{*}(G, W)$ for $p: T \rightarrow W$ a finite étale $G$-map of degree $m$, reduces us to the case of an infinite base-field $k$.

We may replace $Y$ with any open subscheme containing $p_{2}(G \cdot x)$; as this is a finite set of points of $Y$, we may assume that $Y$ is affine; similarly, we may assume that $Y^{\prime}$ is affine. Thus we can factor $f$ as a closed embedding $i: Y^{\prime} \rightarrow \mathbb{A}^{N} \times Y$ followed by the smooth projection $\mathbb{A}^{N} \times Y \rightarrow Y$. This reduces us to the case of 
a closed embedding of affine schemes in $\mathbf{S m} / k$. By a similar argument, we may assume that $X$ is affine.

If $i: Y^{\prime} \rightarrow Y$ is such a closed embedding, of codimension say $q$ then we can factor $i$ as a sequence of $d$ codimension one closed embeddings

$$
Y^{\prime}=Y_{0} \rightarrow Y_{1} \rightarrow \ldots \rightarrow Y_{q-1} \rightarrow Y_{q}=Y
$$

with each $Y_{i}$ smooth in a neighborhood of $p_{2}(G \cdot y)$. By replacing $Y$ with a suitable neighborhood of $p_{2}(G \cdot y)$, we may assume we have a factorization as above with each $Y_{i}$ smooth over $k$. This reduces us to the case of a codimension one closed embedding of smooth affine $k$-schemes.

Let $F$ be a $G$-stable closed subset of $\overline{G \cdot x}$ disjoint from $G \cdot x$ and let $\mathcal{F}$ be a coherent $G$-sheaf supported on $F$. Write $F$ as a union $F=F_{0} \cup F_{1}$, where $F_{0}$ is the union of the irreducible components of $F$ which are disjoint from $G \cdot y$, and $F_{1}$ is the union of the remaining components. The localization properties of $G_{*}(G,-)$ yield a Mayer-Vietoris exact sequence

$$
\ldots \rightarrow G_{0}\left(G, F_{0} \cap F_{1}\right) \rightarrow G_{0}\left(G, F_{0}\right) \oplus G_{0}\left(G, F_{1}\right) \rightarrow G_{0}(G, F) \rightarrow 0
$$

so we can write $[\mathcal{F}]=i_{0 *}\left(x_{0}\right)+i_{1 *}\left(x_{1}\right)$ for elements $x_{j} \in G_{0}\left(G, F_{j}\right)$, where $i_{j}$ : $F_{j} \rightarrow F$ are the inclusions. Since it is clear that $(1 \times f)^{*} i_{0 *}\left(x_{0}\right)$ goes to zero in $G_{0}(G, G \cdot y)$, we may assume that $F=F_{1}$, i.e. that $F \subset \overline{G \cdot y}$.

Suppose $\operatorname{dim}_{k} Y=n+1, \operatorname{dim}_{k} Y^{\prime}=n$. Let $S:=p_{2}(G \cdot y) \subset Y^{\prime}$. By loc. cit. there is a morphism $\pi: Y \rightarrow \mathbb{A}_{k}^{n}$ such that

(1) The restriction of $\pi$ to $\bar{\pi}: Y^{\prime} \rightarrow \mathbb{A}^{n}$ is finite.

(2) $\pi$ is smooth on a neighborhood of $S$ in $Y$.

Form the diagram

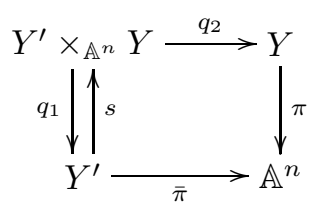

where $s$ is the section to $p_{1}$ induced by the inclusion $Y^{\prime} \rightarrow Y . q_{2}$ is finite. Since $\pi$ is smooth near $S, s\left(Y^{\prime}\right)$ is a Cartier divisor in a neighborhood of $q_{2}^{-1}(S)$. Since $q_{2}$ is finite, there is a neighborhood $V$ of $S$ in $Y$ such that $s(Y)$ is principal on $V^{\prime}:=q_{2}^{-1}(V)$; let $t$ be a defining equation.

Taking the product of (4) with $X$ gives us the diagram of $G$-schemes

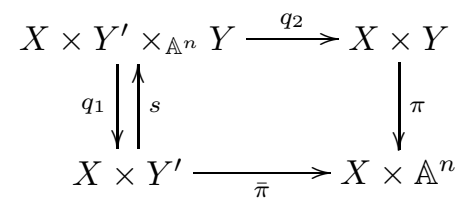

where we omit the $1_{X} \times-$ on the morphisms. $T:=p_{2}^{*}(t)$ is thus a defining equation for $s\left(X \times Y^{\prime}\right)$ over $X \times V^{\prime}$. 
Now set $U:=X \times V, U^{\prime}:=X \times V^{\prime}, D:=s^{-1}\left(s\left(X \times Y^{\prime}\right) \cap U^{\prime}\right) \subset X \times Y^{\prime}$, and let $j: U^{\prime} \rightarrow X \times Y^{\prime} \times \mathbb{A}^{n} Y$ be the inclusion. We have the commutative diagram

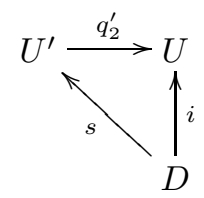

with $s(D)$ defined on $U^{\prime}$ by $T$. Let $\mathcal{G}$ be the restriction of $\mathcal{F}$ to $D$. We have the exact sequence

$$
0 \rightarrow q_{2 *}^{\prime} j^{*} q_{1}^{*} \mathcal{F} \stackrel{\times T}{\longrightarrow} q_{2 *}^{\prime} j^{*} q_{1}^{*} \mathcal{F} \rightarrow i_{*} \mathcal{G} \rightarrow 0
$$

pulling back to $D$ and noting that $i^{*} q_{2 *}^{\prime} j^{*} q_{1}^{*} \mathcal{F}$ is supported in $D \cap \overline{G \cdot y}$ gives the identity in $G_{0}(G, D \cap \overline{G \cdot y})$

$$
i^{*}\left[i_{*} \mathcal{G}\right]=i^{*}\left[q_{2 *}^{\prime} j^{*} q_{1}^{*} \mathcal{F}\right]-i^{*}\left[q_{2 *}^{\prime} j^{*} q_{1}^{*} \mathcal{F}\right]=0
$$

Restricting to $G \cdot y$ completes the proof.

We consider the simplicial set

$$
X_{(p)}^{G}(r):=\left\{[x] \in\left(X \times \Delta^{r}\right)_{(p+r)} / G \mid \overline{G \cdot x} \in S_{(p)}^{G, X}(r)\right\} .
$$

and set

$$
z_{p}(G, X, r):=\bigoplus_{[x] \in X_{(p)}^{G}(r)} K_{0}\left(G_{x}, \operatorname{Spec}(\kappa(x))\right) .
$$

By Proposition 4.1 the cosimplicial structure on $r \mapsto \Delta^{r}$ makes $r \mapsto z_{p}(G, X, r)$ into a simplicial abelian group, denoted $z_{p}\left(G, X,{ }_{-}\right)$.

Definition 4.2. Let $X$ be a finite type $k$-scheme with a $G$-action for a finite group $G$. The equivariant cycle complex of Bredon type, $z_{p}(X, G, *)$, is the complex associated to the simplicial abelian group $z_{p}\left(G, X,{ }_{-}\right)$. Define the equivariant higher Chow groups of Bredon type by

$$
C H_{p}(G, X, r):=\pi_{r}\left(z_{p}\left(G, X,_{-}\right)\right)=H_{r}\left(z_{p}(X, G, *)\right) .
$$

If $X$ is locally equi-dimensional over $k$, we may index by codimension, giving us the simplicial abelian group $z^{p}\left(G, X,{ }_{-}\right)$, the complex $z^{p}(G, X, *)$ and the codimension $p$ equivariant higher Chow groups $C H^{p}(X, G, r)$.

4.2. Functorialities. Let $\rho: L \rightarrow F$ be a finite extension of commutative noetherian rings with $G$-action (compatible via $\rho$ ). $\rho$ induces the exact functor $\rho^{*}$ (restriction of scalars) from the category of finitely generated $F^{t w}[G]$-modules to finitely generated $L^{t w}[G]$-modules and thereby the map on $G$-theories $\rho^{*}: G(G, L) \rightarrow$ $G(G, F)$. For a $k$-algebra homomorphism $\phi: A \rightarrow B$, we have the natural isomorphism of functors

$$
(\rho \otimes \mathrm{id})^{*} \circ(\mathrm{id} \otimes \phi)_{*} \cong(\mathrm{id} \otimes \phi)_{*} \circ(\rho \otimes \mathrm{id})^{*} .
$$

Thus, if $\phi$ has finite Tor-dimension, the diagram

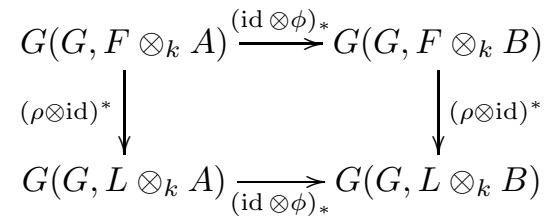


commutes.

If $f: Y \rightarrow X$ is a proper $G$-equivariant morphism of $k$-schemes with $G$-action, we define the push-forward morphism

$$
f_{*}(r): z_{p}(Y, G, r) \rightarrow z_{p}(X, G, r),
$$

by

$$
\left[\alpha \in K_{0}\left(\kappa(Z), G_{z}\right)\right] \mapsto\left[(f \times \mathrm{id})_{*}(\alpha) \in K_{0}\left(\kappa((f \times \mathrm{id})(Z)), G_{(f \times \mathrm{id})(z)}\right)\right]
$$

if $Z \rightarrow(f \times \mathrm{id})(Z)$ is generically finite, and sending $\alpha$ to zero if not. By the commutativity of the above diagram, the maps $f_{*}(r)$ extend to the map of simplicial abelian groups

$$
f_{*}: z_{p}\left(Y, G,{ }_{-}\right) \rightarrow z_{p}\left(X, G,_{-}\right),
$$

with $(f g)_{*}=f_{*} \circ g_{*}$ for proper composable morphisms $f, g$.

Similarly, given a flat $G$-equivariant morphism $f: Y \rightarrow X$ of relative dimension $d$, we have the pullback map

$$
f^{*}: z_{p}\left(X, G,{ }_{-}\right) \rightarrow z_{p+d}\left(Y, G,{ }_{-}\right)
$$

with $(f g)^{*}=g^{*} f^{*}$, and we have the compatibility

$$
g^{*} f_{*}=f_{*}^{\prime} g^{\prime *}
$$

in $G$-equivariant cartesian squares

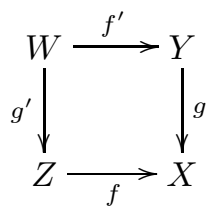

with $f$ proper and $g$ flat.

Remark 4.3. Relying on the localization property of the $z_{p}\left(G, X,,_{-}\right)$, one can extend the contravariant functoriality of $Y \mapsto z^{p}\left(G, X \times_{k} Y,_{-}\right)$from flat morphisms to arbitrary morphisms of smooth $k$-schemes $Y^{\prime} \rightarrow Y$; we will explain this in another paper. However, there seems to be no good pull-back $f^{*}: z^{p}\left(G, X,{ }_{-}\right) \rightarrow z^{p}\left(G, Y,_{-}\right)$, compatible with pull-back on equivariant $G$-theory, for arbitrary $G$-morphisms $f$ : $Y \rightarrow X$, even if both $X$ and $Y$ are smooth over $k$. We give an example of this phenomenon.

Let $k$ be a field, $Z:=\operatorname{Spec} k[x, y], G:=\mathbb{Z} / 2$ and let $G$ act on $Z$ via

$$
(1 \bmod 2 \mathbb{Z}) \cdot(x, y):=(-x,-y) \text {. }
$$

Let $X$ be the line $y=0, Y$ the line $x=0$ and $w$ be the intersection of $X$ and $Y$, i. e. $w=\{(0,0)\}$. Let $y$ be the generic point of $Y$.

We get the following diagram

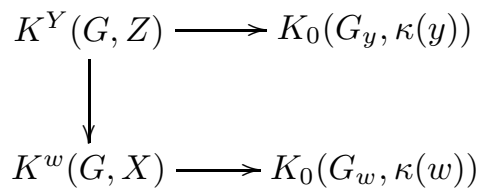

The horizontal arrows are surjective by localization and the bottom horizontal arrow is an isomorphism. The homotopy property for equivariant $K$-theory implies that the vertical arrow is also an isomorphism, again by the localization theorem. 
Since $G_{w}=G$ acts trivially on $\left.\kappa(w), K_{0}\left(G_{w}, \kappa(w)\right)\right)$ is isomorphic to the group ring $\mathbb{Z}[\mathbb{Z} / 2]$. However, since $G=G_{y}$ acts non-trivially on $\kappa(y)$, we have

$$
K_{0}\left(G_{y}, \kappa(y)\right) \cong K_{0}\left(\kappa(y)^{G}\right) \cong \mathbb{Z} .
$$

Thus, there is no arrow on the right which makes this diagram commutative.

This is related to the fact that the ring struction on $K_{0}(X, G)$ (given by tensor product over $\mathcal{O}_{X}$ ) does not in general respect the topological filtration, and so one should not expect the equivariant Chow groups $C H^{*}(G, X, *)$ to have a ring structure. To see an example of this, consider the case $K_{0}(G, X)$, with $X$ and $G$ as above. It follows from the homotopy property and localization that the image of

$$
K_{0}^{w}(G, X) \rightarrow K_{0}(G, X)
$$

is the augmentation ideal of the group ring $\mathbb{Z}[\mathbb{Z} / 2]$. But if $\sigma$ denotes the nonidentity element of $\mathbb{Z} / 2$, we have $(1-\sigma)^{2}=2(1-\sigma)$. Thus, letting $\mathbb{Z}_{\sigma}$ denote the sign representation of $\mathbb{Z} / 2$,

$$
\left(\mathbb{Z}-\mathbb{Z}_{\sigma}\right)^{\otimes 2} \cong 2\left(\mathbb{Z}-\mathbb{Z}_{\sigma}\right)
$$

This shows that $F_{\text {top }}^{1} \cdot F_{\text {top }}^{1} \neq 0$, although $F_{\text {top }}^{2}=0$.

This last point arose during discussions between the first-named author and W. Niziol.

4.3. The cycle map. For each $W \in S_{(p)}^{G, X}(r)$ we have a canonical morphism

$$
\pi_{0}\left(G^{W}\left(G, X \times \Delta^{r}\right)\right) \rightarrow \bigoplus_{[x] \in X_{(p)}^{G}(r), x \in W} K_{0}\left(G_{x}, \operatorname{Spec}(\kappa(x))\right) .
$$

This defines a morphism

$$
\pi_{0}\left(G_{(p)}(G, X, r)\right) \rightarrow \bigoplus_{[x] \in X_{(p)}^{G}(r)} K_{0}\left(G_{x}, \operatorname{Spec}(\kappa(x))\right),
$$

which factors through the surjection

$$
\pi_{0}\left(G_{(p)}(G, X, r)\right) \rightarrow \pi_{0}\left(G_{(p / p-1)}(G, X, r)\right) .
$$

We consider $\pi_{0}\left(G_{(p / p-1)}(G, X, n)\right)$ as a spectrum by using the associated EilenbergMaclane spectrum. Noting that $G_{(p / p-1)}(G, X, n)$ is -1-connected for each $n$, we have the canonical maps of spectra

$$
G_{(p / p-1)}(G, X, n) \rightarrow \pi_{0}\left(G_{(p / p-1)}(G, X, n)\right),
$$

which yield the map of simplicial spectra

$$
G_{(p / p-1)}(G, X,-) \rightarrow \pi_{0}\left(G_{(p / p-1)}(G, X,-)\right) .
$$

We similarly consider $z_{p}\left(G, X,{ }_{-}\right)$as a simplicial spectrum. Taking the composition of the maps described above yields the cycle map

$$
c l_{p}: G_{(p / p-1)}\left(G, X,_{-}\right) \rightarrow z_{p}\left(G, X,,_{-}\right),
$$

defined as a map of simplicial spectra.

Now we can formulate our main result. 
Theorem 4.4. Let $X$ be a scheme of finite type over a field $k$ with an action of a finite group $G$. Suppose that $\frac{1}{\sharp G} \in k$. Then the cycle map

$$
c l_{p}: G_{(p / p-1)}\left(G, X,{ }_{-}\right) \rightarrow z_{p}\left(G, X,{ }_{-}\right)
$$

is a weak equivalence for all $p$.

This and Proposition 3.4 gives

Corollary 4.5. There is a strongly convergent spectral sequence

$$
E_{1}^{p, q}=C H_{p}(G, X,-p-q) \Rightarrow G_{-p-q}(G, X) .
$$

For the proof we first reduce in the next section via localization techniques to the case where $X$ is a point. In the last section we discuss the case of a point.

Remark 4.6. It is easy to see that the cycle map and the spectral sequence are natural with respect to proper pushforward and flat pullback.

\section{LOCALIZATION AND REDUCTION TO THE POINT}

The main result of this section is

Theorem 5.1. Let $G$ be a finite group acting on a finite type $k$-scheme $X, i: W \rightarrow$ $X$ a $G$-stable closed subscheme with open complement $j: U \rightarrow X$. Then for each $p$, the sequence of simplicial spectra

$$
G_{(p)}\left(G, W,_{-}\right) \stackrel{i_{*}}{\rightarrow} G_{(p)}\left(G, X,,_{-}\right) \stackrel{j^{*}}{\longrightarrow} G_{(p)}\left(G, U,_{-}\right)
$$

is a weak homotopy fiber sequence, and the sequence of complexes

$$
z_{p}(G, W, *) \stackrel{i_{*}}{\rightarrow} z_{p}(G, X, *) \stackrel{j^{*}}{\rightarrow} z_{p}(G, U, *)
$$

is isomorphic to a cone sequence in the derived category, i.e., the induced map

$$
\left(j^{*}, 0\right): \operatorname{cone}\left(i_{*}\right) \rightarrow z_{p}(G, U, *)
$$

is a quasi-isomorphism.

Proof. We first consider the $G$-theory sequence. The proof is the same as the proof of the analogous localization theorem in the non-equivariant case Lev01 Cor. 8.2], replacing the spectra $G_{(p)}\left(?,{ }_{-}\right)$with the $G$-equivariant versions $G_{(p)}(G, ?,-)$ throughout. For the readers convenience, we give a sketch of the argument.

Let $S_{(p)}^{G, U^{X}}(r)$ be the subset of $S_{(p)}^{G, U}(r)$ consisting of those $W \subset U \times \Delta^{r}$ with closure $\bar{W} \subset X \times \Delta^{r}$ in $S_{(p)}^{G, U}(r)$. These form a simplicial subset of $r \mapsto S_{(p)}^{G, U}(r)$; let

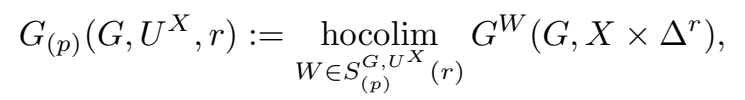

forming the simplicial spectrum $G_{(p)}\left(G, U^{X},{ }_{-}\right)$. The inclusions $S_{(p)}^{G, U^{X}}(r) \subset S_{(p)}^{G, U}(r)$ induce the map of simplicial spectra

$$
\iota: G_{(p)}\left(G, U^{X},{ }_{-}\right) \rightarrow G_{(p)}\left(G, U,_{-}\right)
$$

the localization sequence of $G$-equivariant $G$-theory gives the weak homotopy fiber sequence

$$
G_{(p)}(G, W, r) \stackrel{i_{*}}{\rightarrow} G_{(p)}(G, X, r) \stackrel{j^{*}}{\rightarrow} G_{(p)}\left(G, U^{X}, r\right)
$$


for each $r$, and thus the weak homotopy fiber sequence

$$
G_{(p)}\left(G, W,_{-}\right) \stackrel{i_{*}}{\rightarrow} G_{(p)}\left(G, X,_{-}\right) \stackrel{j^{*}}{\rightarrow} G_{(p)}\left(G, U^{X},_{-}\right) .
$$

Thus, we must show that $\iota$ is a weak equivalence. Since both simplicial spectra are -1-connected, the Hurewicz theorem tells us that it suffices to show that $\iota$ is a homology isomorphism.

For a spectrum $E$, let $\mathbb{Z} E$ be a functorial model for a chain complex with homology groups the homology of $E$. In particular, for $W \subset U \times \Delta^{r}$, we have the complex $\mathbb{Z} G^{W}\left(G, U \times \Delta^{r}\right)$ representing the homology of $G^{W}\left(G, U \times \Delta^{r}\right)$. Taking the limit of $W \in S_{(p)}^{G, U}(r)$ or in $S_{(p)}^{G, U^{X}}(r)$ gives us the complexes $\mathbb{Z} G_{(p)}(G, U, r)$ and $\mathbb{Z} G_{(p)}\left(G, U^{X}, r\right)$ computing the homology of $G_{(p)}(G, U, r)$ and $G_{(p)}\left(G, U^{X}, r\right)$.

For $W \subset U \times \Delta^{r}$, let $W_{n} \subset U \times \Delta^{n}$ be the union of (id $\left.\times g\right)^{-1}(W)$, as $g: \Delta^{n} \rightarrow \Delta^{r}$ runs over structure morphisms for the cosimplicial scheme $\Delta$. Using the usual alternating sum of the pullback by coface maps id $\times \delta_{i}^{r}: U \times \Delta^{r} \rightarrow U \times \Delta^{r+1}$, we form the double complex $n \mapsto \mathbb{Z} G^{W_{n}}\left(G, U \times \Delta^{n}\right)$ and denote the associated total complex by $\mathbb{Z} G^{W}\left(\mathbb{Z} U \times \Delta^{*}\right)$. Thus the limit of the complexes $\mathbb{Z} G^{W}\left(\mathbb{Z} U \times \Delta^{*}\right)$ over $W \in S_{(p)}^{G, U}(r)$ or in $S_{(p)}^{G, U^{X}}(r), r=1,2, \ldots$, computes the homology of $G_{(p)}\left(G, U,{ }_{-}\right)$ and $G_{(p)}\left(G, U^{X}{ }_{-}\right)$. We denote the limits of these complexes by $\mathbb{Z} G_{(p)}(G, U)^{*}$ and $\mathbb{Z} G_{(p)}\left(G, U^{X}\right)^{*}$, respectively. It thus suffices to show that

$$
\iota_{\mathbb{Z}}: \mathbb{Z} G_{(p)}\left(G, U^{X}\right)^{*} \rightarrow \mathbb{Z} G_{(p)}(G, U)^{*}
$$

is a quasi-isomorphism.

$$
\begin{aligned}
& \text { For } W \in S_{(p)}^{G, U}(r), W^{\prime} \in S_{(p)}^{G, U^{X}}(r) \text {, let } \\
& \qquad \iota_{W}: \mathbb{Z} G^{W}\left(G, U \times \Delta^{*}\right) \rightarrow \mathbb{Z} G_{(p)}(G, U)^{*}
\end{aligned}
$$

and

be the canonical maps.

$$
\iota_{W^{\prime}}^{X}: \mathbb{Z} G^{W}\left(G, U \times \Delta^{*}\right) \rightarrow \mathbb{Z} G_{(p)}\left(G, U^{X}\right)^{*}
$$

Next, we construct another pair of complexes which approximate $\mathbb{Z} G_{(p)}(G, U)^{*}$ and $\mathbb{Z} G_{(p)}\left(G, U^{X}\right)^{*}$. For this, fix an integer $N \geq 0$. Let $\partial \Delta_{i}^{N} \subset \Delta^{N}$ be the subscheme defined by $t_{i}=0$; for $I \subset\{0, \ldots, N\}$ let $\partial \Delta_{I}^{N}$ be the face $\cap_{i \in I} \partial \Delta_{i}^{N}$. For $I \supset J$, let $i_{J, I}: \Delta_{I}^{N} \rightarrow \Delta_{J}^{N}$ be the inclusion.

Let $\mathbb{Z} \mathbf{S m} / k$ be the additive category generated by $\mathbf{S m} / k$, i.e., for connected $X$, $Y, \operatorname{Hom}_{\mathbb{Z} \mathbf{S m} / k}(X, Y)$ is the free abelian group on the set of morphisms $\operatorname{Hom}_{\mathbf{S m} / k}(X, Y)$, and disjoint union becomes direct sum. We will construct objects in the category of complexes $C(\mathbb{Z} \mathbf{S m} / k)$.

Form the complex $\left(\Delta^{N}, \partial \Delta^{N}\right)^{*}$ be the complex which is $\oplus_{I,|I|=n} \partial \Delta_{I}^{N}$ in degree $-n$, and with differential

$$
d^{-n}:\left(\Delta^{N}, \partial \Delta^{N}\right)^{-n} \rightarrow\left(\Delta^{N}, \partial \Delta^{N}\right)^{-n+1}
$$

given by $d^{-n}:=\prod_{I,|I|=n} d_{I}^{-n}$, where

$$
d_{I}^{-n}: \partial \Delta_{I}^{N} \rightarrow \oplus_{J,|J|=n-1} \partial \Delta_{J}^{N}
$$

is the sum

$$
d_{I}^{-n}:=\sum_{j=1}^{n} i_{I \backslash\left\{i_{j}\right\}, I},
$$

where $I=\left(i_{1}, \ldots, i_{n}\right), i_{1}<\ldots<i_{n}$. 
We also have the complex $\mathbb{Z} \Delta^{*}$, which is $\Delta^{n}$ in degree $n$, with differential the usual alternating sum of coboundary maps.

The identity map on $\Delta^{N}$ extends to a map of complexes

$$
\Phi^{N}: \mathbb{Z} \Delta^{*} \rightarrow\left(\Delta^{N}, \partial \Delta^{N}\right)[-N]
$$

the maps in degree $r<N$ are all $\pm \mathrm{id}_{\Delta^{r}}$. We can take the product of this construction with $U$, giving us the complex $U \times\left(\Delta^{N}, \partial \Delta^{N}\right)$ and the map of complexes

$$
\Phi^{N}: U \times \mathbb{Z} \Delta^{*} \rightarrow U \times\left(\Delta^{N}, \partial \Delta^{N}\right)[-N]
$$

For $W \in S_{(p)}^{G, U}(N)$, form the complex $\mathbb{Z} G^{W}\left(G, U \times\left(\Delta^{N}, \partial \Delta^{N}\right)\right)$ by taking $\oplus_{I,|I|=n} \mathbb{Z} G^{W_{N-n}}\left(G, U \times \Delta^{N-n}\right)$ in degree $-n$, using the differentials in $U \times\left(\Delta^{N}, \partial \Delta^{N}\right)$ to form a double complex and then taking the total complex. We thus have the map of complexes

$$
\Phi_{W}^{N *}: \mathbb{Z} G^{W}\left(G, U \times\left(\Delta^{N}, \partial \Delta^{N}\right)\right)[-N] \rightarrow \mathbb{Z} G^{W}\left(G, U \times \Delta^{*}\right) .
$$

One shows that $\Phi_{W}^{N *}$ induces a homology isomorphism in degrees $<N$ (see Lev01, Lemma 2.6]).

Take $W \in S_{(p)}^{G, U}(N)$. The main result of [Lev01], Theorem 1.9, gives a map of complexes

$$
\Psi_{W}: U \times \mathbb{Z} \Delta^{*} \rightarrow U \times\left(\Delta^{N}, \partial \Delta^{N}\right)[-N]
$$

and a degree - 1 map

$$
H_{W}: U \times \mathbb{Z} \Delta^{*} \rightarrow U \times\left(\Delta^{N}, \partial \Delta^{N}\right)[-N]
$$

with the following properties:

(1) $d H_{W}=\Psi_{W}-\Phi^{N}$.

(2) Write $\Psi_{W}$ as a sum

$$
\Phi_{W}=\sum_{i=0}^{N} \sum_{I, j|I|=i} n_{i j} \psi_{i j I}
$$

with $\psi_{i j I}: \Delta^{N-i} \rightarrow \partial \Delta_{I}^{N}=\Delta^{N-i}$ maps in $\mathbf{S m} / k$. Then $\psi_{i j I}^{-1}\left(W_{N-i}\right)$ is in $S_{(p)}^{G, U^{X}}(N-i)$

(3) Write $H_{W}$ as a sum

$$
H_{W}=\sum_{i=0}^{N} \sum_{I, j|I|=i} n_{i j} H_{i j I}
$$

with $H_{i j I}: \Delta^{N-i+1} \rightarrow \partial \Delta_{I}^{N}=\Delta^{N-i}$ maps in $\mathbf{S m} / k$. Then $H_{i j I}^{-1}\left(W_{N-i}\right)$ is in $S_{(p)}^{G, U}(N-i+1)$. If $W^{\prime} \subset W_{N-i}$ is in $S_{(p)}^{G, U^{X}}(N-i)$, then $H_{i j I}^{-1}\left(W^{\prime}\right)$ is in $S_{(p)}^{G, U^{X}}(N-i+1)$.

Thus $\Psi_{W}$ induces the map of complexes

$$
\Psi_{W}^{*}: \mathbb{Z} G^{W}\left(G, U \times\left(\Delta^{N}, \partial \Delta^{N}\right)\right)[-N] \rightarrow \mathbb{Z} G_{(p)}\left(G, U^{X}\right)^{*}
$$

and $H_{W}$ gives a degree 1 map

$$
H_{W}^{*}: \mathbb{Z} G^{W}\left(G, U \times\left(\Delta^{N}, \partial \Delta^{N}\right)\right)[-N] \rightarrow \mathbb{Z} G_{(p)}(G, U)^{*}
$$

with

$$
d H_{W}^{*}=\iota_{\mathbb{Z}} \circ \Psi_{W}^{*}-\iota_{W} \circ \Phi_{W}^{N *}
$$


Furthermore, if $W^{\prime} \subset W$ is in $S_{(p)}^{G, U^{X}}(N)$, then $H_{W}$ gives a degree 1 map

$$
H_{W}^{X *}: \mathbb{Z} G^{W^{\prime}}\left(G, U \times\left(\Delta^{N}, \partial \Delta^{N}\right)\right)[-N] \rightarrow \mathbb{Z} G_{(p)}\left(G, U^{X}\right)^{*}
$$

with

$$
d H_{W}^{X *}=\Psi_{W}^{*}-\iota_{W^{\prime}}^{X} \circ \Phi_{W^{\prime}}^{N *}
$$

Since $\Phi_{W}^{N *}$ is a homology isomorphism in degrees $<N$ and $\mathbb{Z} G_{(p)}(G, U)^{*}$ and $\mathbb{Z} G_{(p)}\left(G, U^{X}\right)^{*}$ are the limits of $\mathbb{Z} G^{W}\left(G, U \times \Delta^{*}\right)$ and $\mathbb{Z} G^{W^{\prime}}\left(G, U \times \Delta^{*}\right)$, respectively, this shows that $\iota_{\mathbb{Z}}$ is a quasi-isomorphism, completing the proof.

For the sequence of cycle complexes, let $z_{p}\left(G, U^{X}, r\right)$ be the subgroup of $z_{p}(G, U, r)$ generated by the irreducible codimension $p$ closed subsets $W \subset U \times \Delta^{r}$ with $W \in S_{(p)}^{G, U^{X}}(r)$. This forms the subcomplex $z_{p}\left(G, U^{X}, *\right)$ of $z_{p}(G, U, *)$ and gives us the term-wise exact sequence of complexes

$$
0 \rightarrow z_{p}(G, W, *) \stackrel{i_{*}}{\rightarrow} z_{p}(G, X, *) \stackrel{j^{*}}{\rightarrow} z_{p}\left(G, U^{X}, *\right) \rightarrow 0 .
$$

Thus, we need to show that the inclusion

$$
z_{p}\left(G, U^{X}, *\right) \rightarrow z_{p}(G, U, *)
$$

is a quasi-isomorphism. The proof is now exactly the same as the case of $G$-theory, except that we can avoid the use of the Hurewicz theorem by working directly with the complexes $z_{p}(G, ?, *)$ instead of passing to complexes representing the homology of the simplicial abelian group $z_{p}\left(G, ?,{ }_{-}\right)$.

Corollary 5.2. With the hypotheses and notations as in Theorem [5.1, the sequence

$$
G_{(p / p-1)}\left(G, W,_{-}\right) \stackrel{i_{*}}{\longrightarrow} G_{(p / p-1)}\left(G, X,_{-}\right) \stackrel{j^{*}}{\rightarrow} G_{(p / p-1)}\left(G, U,,_{-}\right)
$$

is a weak homotopy fiber sequence for all $p$.

Proof. This follows directly from Theorem 5.1 the naturality of $i_{*}$ and $j^{*}$ with respect to change of $p$, and the Quetzalcoatl lemma.

Corollary 5.3. Suppose that Theorem 4.4 is true for all fields $k$ with $\frac{1}{\sharp G} \in k$ and with $X=\operatorname{Spec} K$, where $K$ is a finite extension of $k$ with a $G$-action such that $G$ acts trivially on $k$. Then Theorem 4.4 is true for all all fields $k$ with $\frac{1}{\sharp G} \in k$.

Proof. We prove Theorem 4.4 by induction on $\operatorname{dim}_{k} X$, the case of dimension 0 being true by hypothesis.

We have already remarked that the cycle map

$$
c l_{p}: G_{(p / p-1)}\left(G, X,_{-}\right) \rightarrow z_{p}\left(G, X,_{-}\right)
$$

is natural with respect to proper pushforward and pullback with respect to flat maps. Thus, for each $G$-stable closed subset $W \subset X$, we have the commutative diagram

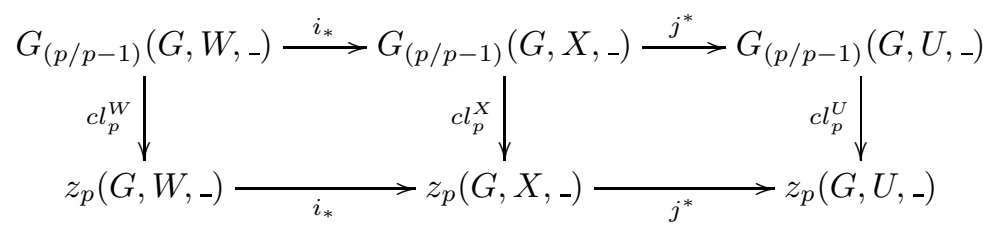

By Theorem 5.1 and Corollary 5.1 the rows are weak homotopy fiber sequences; by our induction hypothesis, $c l_{p}^{W}$ is a weak equivalence, so $c l_{p}^{X}$ is a weak equivalence 
if and only if $c l_{p}^{U}$ is. Taking the limit over all open dense $G$-stable $U \subset X$ reduces us to showing that

$$
c l_{p}^{\kappa(X)}: G_{(p)}\left(G, \kappa(X),{ }_{-}\right) \rightarrow z_{p}\left(G, \kappa(X),{ }_{-}\right)
$$

is a weak equivalence. Breaking up $\kappa(X)$ into a product of fields reduces us to the case of irreducible $X$.

Note that $G_{(p)}\left(G, \kappa(X),{ }_{-}\right), z_{p}\left(G, \kappa(X),{ }_{-}\right)$and $c l_{p}$ do not depend on the choice of "constants" $k \subset \kappa(X)$, so we may replace $k$ with the invariant subfield $\kappa(X)^{G}$. Since the extension $\kappa(X)^{G} \subset \kappa(X)$ is finite, $c l_{p}^{\kappa(X)}$ is a weak equivalence by hypothesis, completing the proof.

\section{THE CASE OF THE POINT}

Now we consider the case of a point. Let $X=\operatorname{Spec}(K), K \supset k$ a finite field extension of $k$ with a $G$-action, with $\frac{1}{\sharp G} \in K$ such that $G$ acts trivially on $k$. Let $\hat{k}:=K^{G}$ be the subfield of $K$ which is fixed under the operation of $G$. Then the field extension $K / \hat{k}$ is finite and Galois; as in the proof of Corollary [5.3, we may replace $k$ with $\hat{k}$. Changing notation, we assume that $k$ is the fixed subfield of $K$ under $G$. We consider the following functor:

$$
\begin{array}{clc}
E: \mathbf{S m} / k^{o p} & \rightarrow & \mathbf{S p t} \\
Y & \mapsto & K\left(G, Y \otimes_{k} K\right)
\end{array}
$$

This clearly defines a presheaf of spectra on $\mathbf{S m} / k$.

We want to show that this presheaf satisfies the axioms of Lev05. We first recall some notations from Lev05.

Let $v(n):=\left\{v_{0}(n), \ldots, v_{n}(n)\right\}$ be the vertices of $\Delta^{n}$, where $v_{i}(n)$ is the point $t_{j}=0, j \neq i, t_{i}=1$. For a field $F$, let $\mathcal{O}_{\Delta_{F}^{n}, v(n)}$ be the semi-local ring of $v(n)$ in $\Delta_{F}^{n}$ and set $\Delta_{0, F}^{n}:=\operatorname{Spec} \mathcal{O}_{\Delta_{F}^{n}, v(n)}$. The $\Delta_{0, F}^{n}$ form a cosimplicial subscheme of $n \mapsto \Delta_{F}^{n}$. We extend this notation to $F$ a product of fields in the evident manner.

We let $\partial_{i} \Delta_{0, F}^{n}:=\partial_{i} \Delta_{F}^{n} \cap \Delta_{0, F}^{n}$, i.e. $\partial_{i} \Delta_{0, F}^{n}$ is the closed subscheme of $\partial \Delta_{0, F}^{n}$ defined by $t_{i}=0$, and set $\partial \Delta_{0, F}^{n}:=\cup_{i=0}^{n} \partial_{i} \Delta_{0, F}^{n}$. We let $\partial_{*} \Delta_{0, F}^{n}$ denote the set of components of $\partial \Delta_{0, F}^{n}$,

$$
\partial_{*} \Delta_{0, F}^{n}:=\left\{\partial_{0} \Delta_{0, F}^{n}, \ldots, \partial_{n} \Delta_{0, F}^{n}\right\}
$$

and denote, e.g., the relative $K$-theory $K\left(\Delta_{0, F}^{n} ; \partial_{0} \Delta_{0, F}^{n}, \ldots, \partial_{n} \Delta_{0, F}^{n}\right)$ by $K\left(\Delta_{0, F}^{n} ; \partial_{*} \Delta_{0, F}^{n}\right)$.

Let $E: \mathbf{S m} / k^{\text {op }} \rightarrow \mathbf{S p t}$ be presheaf of spectra on $\mathbf{S m} / k$. We define the presheaf of spectra $\Omega_{T} E: \mathbf{S m} / k^{\text {op }} \rightarrow$ Spt by

$$
\Omega_{T} E(X):=E^{X \times 0}\left(X \times \mathbb{A}^{1}\right) .
$$

From [Lev05, Section 6], we have the notion of a well-connected functor $E$ : $\mathbf{S m} / k^{\text {op }} \rightarrow$ Spt. From the definition [Lev05, Definition 6.1.1] and Lev05, Proposition 6.3.3], $E$ is well-connected if $E$ satisfies:

(1) Homotopy invariance:

For each $X \in \mathbf{S m} / k$ the map $p^{*}: E(X) \rightarrow E\left(\mathbb{A}^{1} \times X\right)$ is an weak equivalence.

(2) Nisnevich excision:

Let $f: X^{\prime} \rightarrow X$ be an étale morphism in $S m / k$, and let $W \subset X$ be a closed subset. Suppose that $f$ restricts to an isomorphism $f^{-1}(W) \rightarrow W$. Then

$$
f^{*}: E^{W}(X) \rightarrow E^{f^{-1}(W)}\left(X^{\prime}\right)
$$


is a weak equivalence.

(3) Finite descent:

Suppose the base-field $k$ is finite. Let $k^{\prime} \supset k$ be a finite Galois extension of $k$ with group $H$. Consider the presheaf $E_{k^{\prime}}$ on $\mathbf{S m} / k$ defined by $E_{k^{\prime}}(Y):=E\left(Y \times_{k} k^{\prime}\right) . H$ acts on $E_{k^{\prime}}$; let $E_{k^{\prime}}^{H}(Y)$ be a functorial model for the $H$-homotopy fixed point spectrum, giving the presheaf $E_{k^{\prime}}^{H}$ and the natural transformation $\theta_{k^{\prime}}: E \rightarrow E_{k^{\prime}}^{H}$. Then $\theta_{k^{\prime}}$ is a weak equivalence after inverting $\sharp H$.

(4) Well-connectedness (1):

For a closed $W$ in a smooth $X$ the spectrum $E^{W}(X)$ is -1 connected.

(5) Well connectedness (2):

For all $n \geq 1, d \geq 0$, and all finite generated fields $F$ over $k$,

$$
\pi_{0}\left[\left(\Omega_{T}^{d} E\right)\left(\Delta_{0, F}^{n}, \partial_{*} \Delta_{0, F}^{n}\right)\right]=0 .
$$

Proposition 6.1. Let $K$ be a field with $G$-action, $k:=K^{G}$. Suppose that $\frac{1}{\sharp G} \in K$. Suppose further that the presheaf $E: S \boldsymbol{m} / k^{o p} \rightarrow \boldsymbol{S p t}$,

$$
E(X):=K\left(G, X \otimes_{k} K\right),
$$

is well-connected. Then for all $X \in \mathbf{S m} / k$, the cycle map

$$
c l_{p}: G_{(p / p-1)}\left(G, X \otimes_{k} K,_{-}\right) \rightarrow z_{p}\left(G, X \otimes_{k} K,_{-}\right)
$$

is a weak equivalence for all $p$.

Proof. We use the notation of [Lev05, Section 5], except that we index with respect to dimension rather than codimension to maintain the conventions used here.

Since $K / k$ is finite galois with galois group $G$ the obvious map

$$
S_{(p)}^{X}(r) \rightarrow S_{(p)}^{G, X \otimes_{k} K}(r)
$$

is a bijection and therefore we can identify

$$
\begin{aligned}
E_{(p)}(X,-) & =G_{(p)}\left(G, X \otimes_{k} K,-\right) \\
E_{(p / p+1)}(X,-) & =G_{(p / p+1)}\left(G, X \otimes_{k} K,-\right) \\
z_{p}(X ; E,-) & =z_{p}\left(G, X \times_{k} K,-\right) .
\end{aligned}
$$

Further the maps $c l_{p}$ are compatible with these identifications.

Since $E$ is well-connected, it follows from [Lev05 Corollary 5.3.2] that for $X \in$ $\mathrm{Sm} / k$, the simplicial spectrum

$$
E_{(p / p-1)}\left(X,,_{-}\right)=G_{(p / p-1)}\left(G, X \otimes_{k} K,_{-}\right)
$$

is weakly equivalent to the simplicial spectrum $E_{(p / p-1)}^{\text {s.l. }}\left(X,{ }_{-}\right)$. In addition, there is for each $n$ a weak equivalence

$$
c l_{p, n}^{\text {s.l. }}: E_{(p / p-1)}^{\text {s.l. }}(X, n) \rightarrow z_{p}(G, X, n)
$$

(more precisely, to the Eilenberg-Maclane spectrum associated to the abelian group $\left.z_{p}(G, X, n)\right)$.

The argument of Lev05, Theorem 6.4.1], repeated word for word, shows that

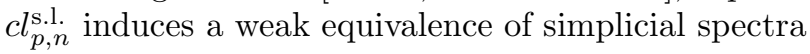

$$
c l_{p}^{\text {s.l. }}: E_{(p / p-1)}^{\text {s.l. }}(X, n) \rightarrow z_{p}(G, X, n)
$$

and that composing $c l_{p}^{\text {s.l. }}$ with the weak equivalence $G_{(p / p-1)}\left(G, X \otimes_{k} K,_{-}\right) \rightarrow$ $E_{(p / p-1)}^{\text {s.l. }}\left(X,{ }_{-}\right)$yields the map $c l_{p}$ (in the stable homotopy category). 
Thus, with the help of Corollary 5.3 to finish the proof of Theorem 4.4 we need only show:

Proposition 6.2. The presheaf $E: S \boldsymbol{S m} / k^{o p} \rightarrow \boldsymbol{S p t}$,

$$
E(Y)=K\left(G, Y \otimes_{k} K\right),
$$

is well-connected.

Proof. We need to show that $E$ satisfies:

(i) Homotopy invariance.

This follows immediately from the homotopy invariance for equivariant $K$ theory.

(ii) Nisnevich excision.

This follows from the fact that the equivariant localization theorem implies $E^{W}(X)=G\left(G, W \otimes_{k} K\right)$.

(iii) Finite descent.

Let $K^{\prime}=K \otimes_{k} k^{\prime}$ and let $\pi: \operatorname{Spec} K^{\prime} \rightarrow \operatorname{Spec} K$ be the projection. Finite descent follows from the existence of the natural pushforward map

$$
\pi_{*}: K\left(G, Y \otimes_{k} K^{\prime}\right) \rightarrow K\left(G, Y \otimes_{k} K\right)
$$

with $\pi_{*} \circ \pi^{*}=\times \sharp H$ on the $K$-groups $K_{n}\left(G, Y \otimes_{k} K\right)$.

(iv) Well-connectedness (1).

Since the equivariant $G$-theory spectrum is -1-connected, $E^{W}(X)$ is -1 connected by the localization property, as in (ii).

(v) Well connectedness (2).

To prove (v), we first note that, since $k \rightarrow K$ is finite and separable, $F \otimes_{k} K$ is a finite product of fields and we have

$$
\begin{aligned}
& \Delta_{0, F}^{n} \times_{k} K \cong \Delta_{0, F \otimes_{k} K}^{n} \\
& \partial_{i} \Delta_{0, F}^{n} \times_{k} K \cong \partial_{i} \Delta_{0, F \otimes_{k} K}^{n}
\end{aligned}
$$

Also, the projective bundle formula for $K\left(G\right.$, , Tho87 shows that $\Omega_{T} K(G$, ) $=$ $K(G$, ). Thus (v) follows from

Lemma 6.3. Let $F$ be a finitely generated field extension of $k$. Then

$$
K_{0}\left(G, \Delta_{0, F \otimes_{k} K}^{n} ; \partial_{*} \Delta_{0, F \otimes_{k} K}^{n}\right)=0 .
$$

Proof. Let $A=F \otimes_{k} K . G$ acts transitively on the irreducible components of $\operatorname{Spec} A$. Fix one component $x:=\operatorname{Spec} \kappa(x)$, and let $G_{x}$ be the isotropy group of $x$. Then

$$
K_{0}\left(G, \Delta_{0, F \otimes_{k} K}^{n} ; \partial_{*} \Delta_{0, F \otimes_{k} K}^{n}\right)=K_{0}\left(G_{x}, \Delta_{0, \kappa(x)}^{n} ; \partial_{*} \Delta_{0, \kappa(x)}^{n}\right) .
$$

In addition, letting $k_{x} \subset K$ be the fixed field of $G_{x}$, we have

$$
F \otimes_{k_{x}} K=\kappa(x) .
$$

Thus, changing notation, we may assume that $F \otimes_{k} K$ is a field $L$ and $F$ is the $G$-fixed subfield of $L$.

We note that $\Delta_{0, L}^{n}$ is an intersection of $G$-stable affine open subschemes $U$ of $\Delta_{L}^{n}$. Thus by Proposition 2.9 the map

$$
\alpha: K_{0}\left(G, \Delta_{0, L}^{n} ; \partial \Delta_{0, L}^{n}\right) \rightarrow K_{0}\left(G, \Delta_{0, L}^{n} ; \partial_{*} \Delta_{0, L}^{n}\right)
$$

is an isomorphism. 
We have the exact sequence

$$
\begin{aligned}
K_{1}\left(G, \Delta_{0, L}^{n}\right) \rightarrow K_{1}\left(G, \partial \Delta_{0, L}^{n}\right) \rightarrow K_{0}\left(G, \Delta_{0, L}^{n} ; \partial \Delta_{0, L}^{n}\right) & \\
& \rightarrow K_{0}\left(G, \Delta_{0, L}^{n}\right) \rightarrow K_{0}\left(G, \partial \Delta_{0, L}^{n}\right)
\end{aligned}
$$

Let $R:=\mathcal{O}_{\Delta_{0, F}^{n}, v(n)}, \bar{R}:=R /\left(\prod_{i=0}^{n} t_{i}\right)$. Since $F \rightarrow L$ is finite, we have

$$
\begin{aligned}
& \Delta_{0, L}^{n}=\operatorname{Spec} R \otimes_{F} L \\
& \partial \Delta_{0, L}^{n}=\operatorname{Spec} \bar{R} \otimes_{F} L
\end{aligned}
$$

Also, by Lemma 2.1

$$
\begin{aligned}
K\left(G, \Delta_{0, L}^{n}\right) & =K\left(R \otimes_{F} L^{t w}[G]\right) \\
K\left(G, \partial \Delta_{0, L}^{n}\right) & =K\left(\bar{R} \otimes_{F} L^{t w}[G]\right)
\end{aligned}
$$

By the lemma below, $R \otimes_{F} L^{t w}[G]$ and $\bar{R} \otimes_{F} L^{t w}[G]$ are semi-local rings, and the surjection $R \otimes_{F} L^{t w}[G] \rightarrow \bar{R} \otimes_{F} L^{t w}[G]$ induces a bijection on the (finite) sets of maximal two-sided ideals. It follows easily from this that $K_{1}\left(R \otimes_{F} L^{t w}[G]\right) \rightarrow$ $K_{1}\left(\bar{R} \otimes_{F} L^{t w}[G]\right)$ is surjective.

Using the notations of the lemma below, we have

$$
\begin{aligned}
& R \otimes_{F} L^{t w}[G]=\prod_{i=1}^{r} M_{n_{i}}\left(R \otimes_{F} D_{i}\right) \\
& \bar{R} \otimes_{F} L^{t w}[G]=\prod_{i=1}^{r} M_{n_{i}}\left(\bar{R} \otimes_{F} D_{i}\right) .
\end{aligned}
$$

In addition, there is a finite separable field extension $F \subset F_{i}$ such that $R \otimes_{F} D_{i}$ is an Azumaya algebra over $R \otimes_{F} F_{i}$ and $\bar{R} \otimes_{F} D_{i}$ is an Azumaya algebra over $\bar{R} \otimes_{F} F_{i}$. Since $R \otimes_{F} F_{i}$ is integral and semi-local, this implies that each projective module over $R \otimes_{F} D_{i}$ is free; by Morita equivalence we have

$$
K_{0}\left(M_{n_{i}}\left(R \otimes_{F} D_{i}\right)\right)=\mathbb{Z},
$$

generated by the class of $\left(R \otimes_{F} D_{i}\right)^{n_{i}}$. This easily implies that the map

$$
K_{0}\left(M_{n_{i}}\left(R \otimes_{F} D_{i}\right)\right) \rightarrow K_{0}\left(M_{n_{i}}\left(\bar{R} \otimes_{F} D_{i}\right)\right)
$$

is injective, hence $K_{0}\left(R \otimes_{F} L^{t w}[G]\right) \rightarrow K_{0}\left(\bar{R} \otimes_{F} L^{t w}[G]\right)$ is injective.

Thus $K_{0}\left(G, \Delta_{0, L}^{n} ; \partial \Delta_{0, L}^{n}\right)=0$, completing the proof.

Lemma 6.4. Let $L$ be a field with an action of a finite group $G, F \subset L$ the fixed subfield of $L$. Suppose that $\frac{1}{\sharp G} \in F$. Let $R$ be a noetherian commutative reduced semi-local $F$-algebra with the property that for each maximal ideal $m \subset R$ we have $R / m \simeq F$. Then

$$
R \otimes_{F} L^{t w}[G]=\prod_{i=1}^{r} M_{n_{i}}\left(R \otimes_{F} D_{i}\right)
$$

where each $D_{i}$ is a central division algebra over $F_{i}$ for some finite separable field extensions $F \subset F_{i}$, and $M_{n_{i}}$ denotes the $n_{i}$ by $n_{i}$ matrix algebra. In addition, the $D_{i}, F_{i}, n_{i}$ and the integer $r$ are independent of $R$, and the center of $M_{n_{i}}\left(R \otimes_{F} D_{i}\right)$ is $R \otimes_{F} F_{i}$. 
Proof. Since $\frac{1}{\sharp G} \in F, L^{t w}[G]$ is a semi-simple, separable algebra over $F$ containing $F$ in its center. Thus, we have $L^{t w}[G]=\prod_{i=1}^{n} M_{n_{i}}\left(D_{i}\right)$ where $D_{i}$ are division rings containing $F$ in their centers, $n_{i} \in \mathbb{N}$. Let $F_{i}$ be the center of $D_{i}$. Since $F \rightarrow L^{t w}[G]$ is a finite separable extension, each $F_{i}$ is finite and separable over $F$.

We claim that $R \otimes_{F} F_{i}$ is the center of $R \otimes_{F} D_{i}$. Indeed, the center $\mathcal{Z}_{i}$ of $R \otimes_{F} D_{i}$ is a finitely generated projective $R$-module containing $R \otimes_{F} F_{i}$. By our assumption on the maximal ideals of $R$, we have $R / m \otimes_{R} \mathcal{Z}_{i}=F_{i}=R / m \otimes_{R} R \otimes_{F} F_{i}$ for all maximal ideals $m \subset R$, so by Nakayama's lemma, $R \otimes_{F} F_{i}=\mathcal{Z}_{i}$.

This completes the proof of the proposition, and the proof of Theorem 4.4

\section{REFERENCES}

[BL95] S. Bloch and S. Lichtenbaum. A spectral sequence for motivic cohomology. Preprint, 1995.

[Blo86] S. Bloch. Algebraic cycles and higher K-theory. Adv. in Math., 61(3):267-304, 1986.

[EG98] D. Edidin and W. Graham. Equivariant intersection theory. Invent. Math., 131(3):595634, 1998.

[EG00] D. Edidin and W. Graham. Riemann-Roch for equivariant Chow groups. Duke Math. J., 102(3):567-594, 2000.

[FS02] E. Friedlander and A. Suslin. The spectral sequence relating algebraic $K$-theory to motivic cohomology. Ann. Sci. École Norm. Sup, (4) 35(6):773-875, 2002.

[Gro71] A. Grothendieck. Revêtements étales et groupe fondamental. Springer-Verlag, Berlin, 1971. Séminaire de Géométrie Algébrique du Bois Marie 1960-1961 (SGA 1), Dirigé par Alexandre Grothendieck. Augmenté de deux exposés de M. Raynaud, Lecture Notes in Mathematics, Vol. 224.

[Jos05] R. Josuha. Bredon style homology and cohomology for algebraic stacks. Preprint (http://www.math.uiuc.edu/K-theory/0733/), 2005.

[Lev94] M. Levine. Bloch's higher Chow groups revisited. In K-theory (Strasbourg, 1992), volume 226 of Astérisque, pages 235-320. Soc. Math. de France, 1994.

[Lev01] M. Levine. Techniques of localization in the theory of algebraic cycles. J. Alg. Geom., 10:299-363, 2001.

[Lev05] M. Levine. The homotopy coniveau filtration. Preprint (http://arxiv.org/abs/math.AG/0510334), 2005.

[Pan98] R. Pandharipande. Equivariant Chow rings of o $(k)$, so $(2 k+1)$, and so(4). J. Reine Angew. Math., 496:131-148, 1998.

[Qui73] D. Quillen. Higher algebraic K-theory I. In Algebraic K-theory I, volume 341 of Lecture Notes in Math., pages 85-147. Springer Verlag, Berlin, 1973.

[Tho87] R. W. Thomason. Algebraic $K$-theory of group scheme actions. In Algebraic topology and algebraic K-theory (Princeton, N.J., 1983), volume 113 of Ann. of Math. Stud., pages 539-563. Princeton Univ. Press, Princeton, NJ, 1987.

[Tot99] B. Totaro. The Chow ring of a classifying space. In Algebraic K-theory (Seattle, WA, 1997), volume 67 of Proc. Sympos. Pure Math., pages 249-281. Amer. Math. Soc., Providence, RI, 1999.

[TT90] R. W. Thomason and Thomas Trobaugh. Higher algebraic $K$-theory of schemes and of derived categories. In The Grothendieck Festschrift, Vol. III, volume 88 of Progr. Math., pages 247-435. Birkhäuser, Boston, MA, 1990.

[Vor79] T. Vorst. Localization of the $K$-theory of polynomial extensions. Math. Ann., 244(1):3353, 1979. With an appendix by Wilberd van der Kallen.

[Wei89] C. A. Weibel. Homotopy algebraic $K$-theory. In Algebraic K-theory and algebraic number theory (Honolulu, HI, 1987), volume 83 of Contemp. Math., pages 461-488. Amer. Math. Soc., Providence, RI, 1989. 